\title{
Renormalization Group Approach to the Infrared Behavior of a Zero-Temperature Bose System
}

\author{
F. Pistolesi ${ }^{1}$, C. Castellani ${ }^{2}$, C. Di Castro ${ }^{2}$, and G.C. Strinati ${ }^{3}$ \\ ${ }^{1}$ Laboratoire de Physique et Modélisation des Milieux Condensès \\ CNRS-UJF, B.P. 16638042 Grenoble, France \\ ${ }^{2}$ Dipartimento di Fisica, Università "La Sapienza", UdR INFM, I-00185 Roma, Italy \\ ${ }^{3}$ Dipartimento di Fisica, Università di Camerino, UdR INFM, I-62032 Camerino, Italy
}

(November 5, 2018)

\begin{abstract}
We exploit the renormalization-group approach to establish the exact infrared behavior of an interacting Bose system at zero temperature. The local-gauge symmetry in the broken-symmetry phase is implemented through the associated Ward identities, which reduce the number of independent running couplings to a single one. For this coupling the $\epsilon$-expansion can be controlled to all orders in $\epsilon(=3-d)$. For spatial dimensions $1<d \leq 3$ the Bogoliubov fixed point is unstable towards a different fixed point characterized by the divergence of the longitudinal correlation function. The Bogoliubov linear spectrum, however, is found to be independent from the critical behavior of this correlation function, being exactly constrained by Ward identities. The new fixed point properly gives a finite value of the coupling among transverse fluctuations, but due to virtual intermediate longitudinal fluctuations the effective coupling affecting the transverse correlation function flows to zero. As a result, no transverse anomalous dimension is present. This treatment allows us to recover known results for the quantum Bose gas in the context of a unifying framework and also to reveal the non-trivial skeleton structure of its perturbation theory.
\end{abstract}

\section{INTRODUCTION}

Perturbation theory for neutral zero temperature interacting bosons in the superfluid phase has been developed in the fifties ${ }^{1,2}$ following the mean-field result of Bogoliubov. ${ }^{3}$ Notwithstanding the success of its immediate implementation in the low density $\operatorname{limit}^{1}$ and in proving the coincidence of the quasiparticle and sound modes, ${ }^{4-7}$ the unresolved problem of infrared (IR) divergences due to the Goldstone mode led to surprising results. As a matter of fact, much later A. Nepomnyashchii and Y. Nepomnyashchii ${ }^{8}$ (from here on referred as NN) demonstrated that the fundamental quantity $\Sigma_{12}(k)$, the anomalous self-energy, must actually vanish for vanishing momentum $(k \rightarrow 0)$ and $d \leq 3$. In the Bogoliubov approximation the existence of a linear spectrum relies on the presence of $\Sigma_{12}(k) \neq 0$. Since NN found that the linear spectrum is preserved for $k \rightarrow 0$, their result implies that the structure of the quadratic part of the action must be actually much more complicated than the simple Bogoliubov form.

The NN results were obtained by studying directly the skeleton structure of the diagrammatic perturbation theory (PT). To determine the asymptotic behavior of the correlation functions for $k \rightarrow 0 \mathrm{NN}$ had to resort to a self-consistent analysis of the skeleton perturbation theory, finding relations among set of diverging diagrams. This method, besides being not fully transparent, might not be readily controlled. The need of developing a PT free from IR divergences was actually realized by Popov ${ }^{9}$ before NN. Popov development was achieved within a functional-integral approach, by introducing a phase-amplitude representation for the bosonic variables in the IR region for $|\mathbf{k}|$ smaller than a cutoff $k_{o}$. In this way at all intermediate steps the calculation turned out to be free from IR divergences. However, elimination of the arbitrary parameter $k_{o}$ remains nontrivial in Popov approach, as it requires full control of the IR region up to momenta of the order of $k_{o}$ where the phase-amplitude description becomes less and less accurate since at large momenta it is the particle representation to be appropriate. In addition, the phase-amplitude description somewhat obscures the particle viewpoint even at small momenta. As a matter of fact, when $k \rightarrow 0$ it is not easy to follow with the phase-amplitude description the nontrivial evolution of the elementary excitations from free particles to the sound mode. Probably, it was for this reason that Popov recognized in his own language the NN result about the vanishing of the anomalous self-energy only at a later stage. ${ }^{10}$

The vanishing of the anomalous self-energy is not a mere mathematical result but it has a definite physical origin. In this particular context of broken gauge symmetry, this finding reflects the general picture given by Patašinskij and Pokrovskij, ${ }^{11}$ whereby the Goldstonemode singularity of the transverse correlation function drives a divergence also in the longitudinal correlation function for a continuous broken symmetry. An additional interesting issue is to understand how, technically, the IR divergences do not lead to a anomalous dimension for the quadratic propagator, as it happens instead for critical phenomena.

These circumstances lead to the necessity of providing a unifying and fully controlled treatment of IR divergences. In this respect, the renormalization group (RG) approach appears to be the natural and reliable tool to determine the IR behavior of the system in the presence of IR divergences. In this paper, we exploit the RG approach to obtain the exact IR behavior of the 
vertex functions (and thus of the correlation functions) for a neutral system of interacting bosons in the brokensymmetry phase at zero temperature. To this end, we will make extensive use of the Ward identities (WI) associated with gauge invariance, which pose strong constraints on the RG equations and enable us to obtain the desired solution to all orders of the $\epsilon$-expansion (here $\epsilon=3-d)$. Although Popov method might at a first look be preferable to deal with the broken-symmetry phase since it deals at the outset with variables that are manifestly gauge invariant, it appears useful to complement the phase-amplitude approach by studying the IR behavior of the system via a more standard particle representation. Our treatment allows us to follow the evolution of the propagator continuously, from the particle region to the hydrodynamical one. A short account of this work has been given in Ref. 12 .

Usually, the appearance of IR divergences is related to a second-order phase transition, when a competition between two different phases with equal free energy leads to divergent fluctuations at the critical temperature. In the present case, although the system is in a stable phase and no phase transition occurs, there exists a competition among degenerate states which are associated with different values of the macroscopic phase of the order parameter and are thus physically equivalent. This leads to the divergence of the longitudinal correlation function mentioned above, while all correlation functions obtained as averages of local gauge-invariant operators (like the density-density correlation function) are expected to be free from IR divergence. A similar behavior is found for fermionic systems, where the perturbation theory for the stable phase of the Luttinger liquid presents infrared divergences that have to disappear when the symmetry is correctly enforced through Ward identities. ${ }^{13}$

Perturbation theory for the broken symmetry phase in the particle representation considers averages of operators which are not local gauge invariant, and is therefore plagued by IR divergences at intermediate steps of the calculation. It is clear that these divergences cannot be independent from each other as they have to cancel out when calculating averages of the above-mentioned local gauge-invariant operators. In this respect, Ward identities provide explicit connections among the divergences. In turn, this makes all local gauge-invariant susceptibilities (like the compressibilities) finite and stabilizes the system accordingly with respect to phase fluctuations.

By our RG approach we will be able to prove that the three available gauge-invariant susceptibilities of the system (namely, the condensate susceptibility, the ordinary susceptibility related to the sound velocity, and the total density) are indeed finite, being invariants of the RG flow. This will be done by establishing explicit connections among the running couplings of the theory. In this way, we will be left with only one independent running coupling, whose infrared behavior will then be established to all orders in $\epsilon(=3-d)$ for $d>1$. The fixed point for $d<3$ is characterized by a finite inter- action coupling among the transverse fluctuating fields. This at first sight suggests that the theory is interacting at the fixed point like the standard $\phi^{4}$ theory. ${ }^{14} \mathrm{We}$ find, in fact, that this is not true due to the presence of the longitudinal field. One can define an effective interaction among transverse fluctuations entering the relevant subset of diagrams responsible of the appearance of the anomalous dimension in $\phi^{4}$ theory. This effective interaction is flowing to zero at the fixed point, thus preserving the $1 / k^{2}$ behavior for the transverse propagator. The occurrence of the sound spectrum will turn out to be independent from the scaling behavior of the independent running couplings, as it depends only on the underlying gauge symmetry. We will also recover the leading IR behavior of the single-particle Green's functions and of the (two-particle) response functions obtained previously by Gavoret and Nozières ${ }^{4}$ and by $\mathrm{NN}^{8}$.

$\mathrm{RG}$ treatments of the zero-temperature interactingboson problem have been previously given by Weichman $^{15}$ and by Benfatto ${ }^{16}$. Weichman found the correct exponent of the longitudinal susceptibility by performing a one-loop calculation for an intrinsically non-divergent quantity (the free energy) and then used a simple scaling argument to determine the singular behavior of the longitudinal single-particle Green's function. This scaling argument can, in general, be justified by the RG approach, as it will be shown below by our treatment. Benfatto, on the other hand, used a Wilson-like RG approach in a rigorous fashion to determine the scaling behavior for a number of running couplings. However, his treatment was limited to $d=3$ and did not take advantage of a systematic implementation of WI, which in turn would guarantee the linear behavior of the spectrum irrespective of the IR behavior of the longitudinal correlation function. The fact that the theory is asymptotically free in $d=3$, as found by Benfatto, is thus not essential to stabilize the superfluid phase by preserving the linear spectrum.

The question naturally arises whether the momentum region, where phase fluctuations (leading to IR divergences) dominate, has physical relevance apart from establishing the superfluid behavior. To this end, a generalized Ginzburg criterion can be introduced for the momentum variable to determine, in particular, whether the IR region will or will not merge into the Bogoliubov region, where the spectrum is still linear but the longitudinal correlation function is approximately constant. For the low-density Bose gas one can explicitly show ${ }^{10}$ that the Ginzburg region extends up to a characteristic momentum that vanishes exponentially (in the gas parameter) with respect to the extension of the linear Bogoliubov region. In the more general case, it is not a priori possible to assess whether the Bogoliubov region might be washed out by the growing of the Ginzburg region. In this case a full RG approach is required, as shown in the present paper.

The plan of the paper is as follows. In Section II we recall how IR divergences manifest themselves in PT by 
considering the one-loop correction to the Bogoliubov approximation, and then set up the RG treatment for the problem at hand. In Section III we derive and analyze the WI relevant to our purposes. In Section IV we determine the exact IR behavior of the vertex functions in the context of the $\epsilon$ expansion. Section $\mathrm{V}$ gives our conclusions. For completeness, additional technical points are discussed in the Appendices. Specifically, Appendix A deals with the definition of gauge invariance in terms of the couplings introduced in this paper, Appendix B shows the one-loop evaluations of relevant vertex functions and the validity of the WI, Appendix $\mathrm{C}$ gives the one-loop equations for the running couplings entering the propagators.

\section{IR DIVERGENCES AND SETUP OF THE RG TREATMENT}

In this Section, we briefly recall how the IR divergences appear in the PT for an interacting boson system in the presence of a condensate beyond the Bogoliubov approximation, and then set up the RG treatment to heal these divergences. To this end, we will exploit a functional-integral approach which is especially suited to the purpose. ${ }^{17}$

\section{A. Bogoliubov approximation and the appearance of IR divergences}

We consider a system of neutral bosons interacting through a short-range potential $v$, in the presence of auxiliary external sources $\lambda, \mu$, and $\mathbf{A}$ that serve to generate the field, density, and current connected correlation functions. The associated action is given by:

$$
\begin{aligned}
S=\int d x & \left\{\psi^{*}(x)\left[\partial_{\tau}+\mu(x)\right] \psi(x)-|(\nabla-i \mathbf{A}) \psi(x)|^{2}\right. \\
& \left.-\frac{v}{2}|\psi(x)|^{4}+\psi(x) \lambda^{*}(x)+\psi^{*}(x) \lambda(x)\right\}
\end{aligned}
$$

In this expression, $x=(\tau, \mathbf{r}$ ) (with $\tau$ ranging from 0 to the inverse temperature $\beta$ ) is a vector with $d+1$ dimensions, $\psi(x)$ is a bosonic field obeying periodic boundary conditions over the imaginary time $\tau$, and $v$ is the two-body interaction. We shall always consider the zero temperature limit $\beta \rightarrow \infty$. Since we are concerned with the IR behavior, we can choose $v$ local from the outset, with an ultraviolet (UV) cutoff implied in UV divergent integrals. We have also set $\hbar=1, k_{B}=1$, and $m=1 / 2$.

The sources $\lambda(x)$ and $\mathbf{A}(x)$ are let to vanish at the end of the calculation, while $\mu(x)$ reduces to the ordinary chemical potential $\mu$. In the following, we shall sometimes use the short-hand notation $A_{\nu}=(\mu, \mathbf{A})$ with $\nu=0, \ldots, d$.

The relevant correlation functions can be obtained from the free energy functional $F$, defined as:

$$
F\left[\lambda, \lambda^{*}, A_{\nu}\right]=\ln \int \mathcal{D} \psi^{*} \mathcal{D} \psi e^{S\left[\psi, \psi^{*}, \lambda, \lambda^{*}, A_{\nu}\right]}
$$

In particular, the single-particle normal and anomalous Green's functions are given by

$$
\left\{\begin{array}{l}
\mathcal{G}_{11}\left(x_{1}, x_{2}\right) \equiv-\left\langle\psi\left(x_{1}\right) \psi^{*}\left(x_{2}\right)\right\rangle_{c}=-\frac{\delta F}{\delta \lambda^{*}\left(x_{1}\right) \delta \lambda\left(x_{2}\right)} \\
\mathcal{G}_{12}\left(x_{1}, x_{2}\right) \equiv-\left\langle\psi\left(x_{1}\right) \psi\left(x_{2}\right)\right\rangle_{c}=-\frac{\delta F}{\delta \lambda^{*}\left(x_{1}\right) \delta \lambda^{*}\left(x_{2}\right)}
\end{array}\right.
$$

where the connected average $\left\langle\psi^{*} \psi\right\rangle_{c}=\left\langle\psi^{*} \psi\right\rangle-\left\langle\psi^{*}\right\rangle\langle\psi\rangle$ is defined with respect to the weight function $\exp \{S\}$.

Bose condensation is conveniently introduced in terms of the Fourier transforms

$$
\left\{\begin{aligned}
\psi(x) & =(\beta \Omega)^{-1 / 2} \sum_{\omega_{s}, \mathbf{k}} e^{i\left(\omega_{s} \tau+\mathbf{k r}\right)} \psi\left(\omega_{s}, \mathbf{k}\right) \\
\psi^{*}(x) & =(\beta \Omega)^{-1 / 2} \sum_{\omega_{s}, \mathbf{k}} e^{-i\left(\omega_{s} \tau+\mathbf{k r}\right)} \psi^{*}\left(\omega_{s}, \mathbf{k}\right)
\end{aligned}\right.
$$

where $\mathbf{k}$ is a wave vector, $\omega_{s}=2 \pi s \beta^{-1}$ ( $\mathrm{s}$ integer) is a bosonic Matsubara frequency, and $\Omega$ is the volume occupied by the system. We set

$$
\left\{\begin{array}{rl}
\psi(k) & =\tilde{\psi}(k)+(\beta \Omega)^{1 / 2} \delta_{k, 0} \alpha \\
\psi^{*}(k) & =\tilde{\psi}^{*}(k)+(\beta \Omega)^{1 / 2} \delta_{k, 0} \alpha^{*}
\end{array},\right.
$$

where now $k=\left(\omega_{s}, \mathbf{k}\right)$ and $\alpha$ is arbitrary, and introduce Eq. (2.4) into the action (2.1). In this way, the action can be split into a (free) quadratic part $S_{Q}=$ $\sum_{k} \tilde{\psi}^{*}(k) \tilde{\psi}(k) \mathcal{G}_{o}^{-1}(k)$ with associated inverse propagator

$$
\mathcal{G}_{o}^{-1}(k)=i \omega_{s}-\mathbf{k}^{2}+\mu
$$

and into the remaining perturbation terms. ${ }^{1}$ The parameter $\alpha$ is then fixed by requiring that $\langle\tilde{\psi}(k=0)\rangle=0$, which corresponds to the absence of "tadpole" diagrams, ${ }^{9}$ i.e., to the vanishing of 1-particle irreducible diagrams with a single line entering:

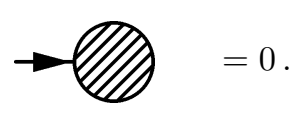

The value of $\alpha$ thus becomes a function of the uniform external source $\lambda$, and coincides with the (square root of the) condensate density in the physical limit $\lambda \rightarrow 0$. This requirement simplifies PT considerably. In particular, it is convenient to introduce a matrix Green function by defining $\psi_{1}(k)=\tilde{\psi}(k)$ and $\psi_{2}(k)=\tilde{\psi}^{*}(-k)$. In this way, $\mathcal{G}_{11}(k)=\mathcal{G}_{22}^{*}(-k)=-\left\langle\tilde{\psi}(k) \tilde{\psi}^{*}(k)\right\rangle$ and $\mathcal{G}_{12}=\mathcal{G}_{21}^{*}(-k)=-\langle\tilde{\psi}(k) \tilde{\psi}(-k)\rangle$ satisfy the DysonBeliaev equation $\mathcal{G}(k)^{-1}=G_{o}^{-1}(k)-\Sigma(k)$ with

$$
G_{o}=\left(\begin{array}{cc}
\mathcal{G}_{o}(k) & 0 \\
0 & \mathcal{G}_{o}(-k)
\end{array}\right)
$$


and the matrix elements of the self-energy $\Sigma$ are distinguished by the number of incoming and outgoing lines. The off-diagonal terms $\Sigma_{12}(k)=\Sigma_{21}^{*}(k)$ characterize the broken-symmetry state as they vanish identically in the normal state. Given $\Sigma(k)$, a simple matrix inversion leads to $\mathcal{G}$. The Bogoliubov approximation consists in taking the diagrams with no loops for the self-energy: $\Sigma_{11}(k)=2 v|\alpha|^{2}, \Sigma_{12}(k)=\alpha^{2} v$. Within the same approximation, the vanishing of tadpole diagrams is equivalent to the vanishing of the linear term in the action. This gives for $\lambda=0$ :

$$
v|\alpha|^{2}=\mu
$$

and the following form for the Green's functions:

$$
\mathcal{G}_{11}(k)=-\frac{i \omega_{s}+\mathbf{k}^{2}+\mu}{\omega_{s}^{2}+E_{\mathbf{k}}^{2}} \quad \mathcal{G}_{12}(k)=\frac{\mu}{\omega_{s}^{2}+E_{\mathbf{k}}^{2}}
$$

with $E_{\mathbf{k}}^{2}=\mathbf{k}^{4}+2 \mu \mathbf{k}^{2}$. Note that, within the Bogoliubov approximation, the occurrence of the sound mode (i.e., $E_{\mathbf{k}} \propto|\mathbf{k}|$ for $\left.\mathbf{k}^{2} \ll 2 \mu\right)$ is strictly related to $\Sigma_{12}(k=0)$ being non-vanishing.

Inclusion of higher-order terms beyond the Bogoliubov approximation, however, spoils the above results due to the occurrence of IR divergences. For instance, let us consider the following one-loop diagram contributing to $\Sigma_{12}$ :

$$
A(k)=\rightarrow \bigcap_{-k}^{k+q}-\sim \alpha^{2} v^{2} c_{o} \int^{\Lambda} \frac{d^{d+1} \tilde{q}}{\tilde{q}^{2}(\tilde{k}+\tilde{q})^{2}}
$$

where $\Lambda$ is an UV cutoff and $\tilde{q}$ is a $(d+1)$-dimensional vector. We have used the small $k$-form of the Bogoliubov propagators (2.9) that behave behave like $\tilde{k}^{-2}$, where $\tilde{k}=\left(\omega_{s} / c_{o}, \mathbf{k}\right)$ with $c_{o}^{2}=2 \mu$ being the square of the sound velocity. Problems arise because $A(k)$ diverges when $d \leq 3$ as $k \rightarrow 0$ (in the zero-temperature limit) as follows:

$$
A(k) \sim \begin{cases}k^{d-3} & \text { for } d<3 \\ \ln \left(k^{2} / \Lambda^{2}\right) & \text { for } d=3\end{cases}
$$

while it remains finite for $d>3$. The one-loop contribution (2.10) thus overcomes the Bogoliubov contribution for small enough $k$. It is then natural to introduce in this context a generalized Ginzburg criterion for the momentum variable, by comparing one-loop and zero-loop self-energies:

$$
1 \approx \frac{\Sigma_{12}^{(1)}\left(k_{G}\right)}{\Sigma_{12}^{(0)}\left(k_{G}\right)}=\left(v^{3} n\right)^{1 / 2} \begin{cases}k_{G}^{d-3} & d<3 \\ \log \left(c_{o} / k_{G}\right) & d=3 .\end{cases}
$$

Here, we have used $\alpha^{2} \approx n$ and we set the UV cutoff $\Lambda$ equal to $c_{o}$ as simple estimates. According to this criterion, IR divergences become dangerous when $k \lesssim k_{G}$, where $k_{G}=\left(v^{3} n\right)^{\frac{1}{2(3-d)}}$ for $d<3$ and $k_{G}=$ $c_{o} \exp \left\{-\left(v^{3} n\right)^{-1 / 2}\right\}$ for $d=3$ as given in Ref. 10 .

Notwithstanding the presence of these singularities, explicit calculations have shown that IR divergences cancel out in most physical quantities. ${ }^{1}$ A notable example is the Hugenholtz-Pines identity ${ }^{2}$, namely,

$$
\Sigma_{11}(k=0)-\Sigma_{12}(k=0)=\mu
$$

which implies cancellation of the IR divergences in the two self-energies.

The identity (2.13) provides the simplest example of WI, whereby the (irreducible) diagrams of PT are constrained by the underlying gauge symmetry. A systematic study of the implications of WI was originally performed in Ref. ${ }^{4}$. In the following Sections we will exploit extensively the whole set of WI to reduce the set of IR divergent diagrams of PT to only a few independent ones.

As mentioned in the Introduction, not all IR divergences, however, disappear from the theory. In particular, the longitudinal single-particle Green's function (to be discussed in detail in Section IV) has to diverge in the IR on physical grounds, ${ }^{11,18}$ implying that

$$
\Sigma_{12}(k=0)=0
$$

for $d \leq 3$. This was found as an exact result by NN. It is clear that the result (2.14) which depends on dimensionality cannot be inferred from a WI like Eq. (2.13), which is instead independent from dimensionality. It is also clear that WI by themselves are not sufficient to specify completely the IR behavior of the system. To this purpose, specific dynamical properties have to be taken into account in addition to WI, as it will be discussed in Section IV where the RG equation for a single independent running coupling will be derived.

\section{B. Representation for the Field-Theory RG treatment of interacting bosons}

The RG treatment in the broken-symmetry phase is considerably simplified when replacing the Bose field $\psi$ and $\psi^{*}$ by their longitudinal $\left(\psi_{l}\right)$ and transverse $\left(\psi_{t}\right)$ components to the direction of the broken symmetry: ${ }^{16}$

$$
\left\{\begin{aligned}
\psi(x) & =\alpha+\tilde{\psi}_{l}(x)+i \tilde{\psi}_{t}(x) \\
\psi^{*}(x) & =\alpha+\tilde{\psi}_{l}(x)-i \tilde{\psi}_{t}(x)
\end{aligned}\right.
$$

where $\tilde{\psi}_{l}$ and $\tilde{\psi}_{t}$ are real functions, and the order parameter has been taken real as well without loss of generality. These new fields are introduced to distinguish explicitly between transverse and longitudinal fluctuations, whose difference is not evidenced by the standard representation resulting into Eq. (2.9). 
In terms of Fourier transform of the new fields, the action (2.1) becomes for $\mathbf{A}=\mathbf{0}$ and $\mu(x)=\mu$ :

$$
\begin{aligned}
& S\left[\tilde{\psi}_{i}\right]=\beta \Omega\left[\mu \alpha^{2}-\frac{v}{2} \alpha^{4}+\lambda_{l} \alpha\right]+(\beta \Omega)^{1 / 2}\left[2 \mu \alpha-2 v \alpha^{3}+\lambda_{l}\right] \tilde{\psi}_{l}(0)+(\beta \Omega)^{1 / 2} \lambda_{t} \tilde{\psi}_{t}(0) \\
& +\sum_{k}\left\{\left[\mu-3 v \alpha^{2}-\mathbf{k}^{2}\right] \tilde{\psi}_{l}(k) \tilde{\psi}_{l}(-k)+\left[\mu-v \alpha^{2}-\mathbf{k}^{2}\right] \tilde{\psi}_{t}(k) \tilde{\psi}_{t}(-k)-2 \omega_{\nu} \tilde{\psi}_{l}(-k) \tilde{\psi}_{t}(k)\right\} \\
& -2 v \alpha(\beta \Omega)^{-1 / 2} \sum_{k_{1}+k_{2}+k_{3}=0}\left\{\tilde{\psi}_{l}\left(k_{1}\right) \tilde{\psi}_{l}\left(k_{2}\right) \tilde{\psi}_{l}\left(k_{3}\right)+\tilde{\psi}_{l}\left(k_{1}\right) \tilde{\psi}_{t}\left(k_{2}\right) \tilde{\psi}_{t}\left(k_{3}\right)\right\} \\
& -(\beta \Omega)^{-1} \sum_{k_{1}+k_{2}+k_{3}+k_{4}=0} \frac{v}{2}\left\{\tilde{\psi}_{l}\left(k_{1}\right) \tilde{\psi}_{l}\left(k_{2}\right) \tilde{\psi}_{l}\left(k_{3}\right) \tilde{\psi}_{l}\left(k_{4}\right)+\tilde{\psi}_{t}\left(k_{1}\right) \tilde{\psi}_{t}\left(k_{2}\right) \tilde{\psi}_{t}\left(k_{3}\right) \tilde{\psi}_{t}\left(k_{4}\right)+2 \tilde{\psi}_{l}\left(k_{1}\right) \tilde{\psi}_{l}\left(k_{2}\right) \tilde{\psi}_{t}\left(k_{3}\right) \tilde{\psi}_{t}\left(k_{4}\right)\right\}
\end{aligned}
$$

Quite generally, the order parameter is given by

$$
\psi_{i o}(x) \equiv\left\langle\psi_{i}(x)\right\rangle=\delta F / \delta \lambda_{i}(x)
$$

where $i=(l, t), \lambda=\left(\lambda_{l}+i \lambda_{t}\right) / 2$, and $F$ is given by Eq. (2.2) (once expressed in terms of $\lambda_{l}$ and $\lambda_{t}$ ). Consistently with our choice, we shall eventually assume the limit $\lambda_{t}=0$ and $\lambda_{l} \rightarrow 0$, so that only $\psi_{l o}$ survives.

The variable $\alpha$ can be eliminated by the tadpole condition. It can be useful to clarify this point in more details here, since at higher orders elimination of $\alpha$ becomes more subtle. Given the action (2.16), one can regard the quadratic part as the free theory and treat the remainder as a perturbation, including the linear term in $\tilde{\psi}_{l}$ (we set $\lambda_{i}=0$ from the outset). The diagrammatic perturbation theory is then well defined. Diagrams are conveniently summed up by grouping them according to the number of loops present in each of them. One can verify that this is equivalent to scale the action as $S \rightarrow S / a$ and calculate all quantities by an expansion in the parameter $a$. A naive way of proceeding would be to choose the value of $\alpha$ such that the linear term in the action vanishes, giving $\alpha^{2} v=\mu$. In this way, propagators are massless and all physical quantities can be calculated within the loopexpansion with the parameter $\alpha$ fixed to its mean-field value $\sqrt{\mu / v}$. Actually, the presence of the 3-legs vertices makes this approach cumbersome. As a matter of fact, all diagrams will have tadpole corrections. It is not difficult to recognize that the effect of these corrections could be incorporated in a redefinition of $\alpha$. The shift required would be exactly the correction to the average of $\psi_{l}$ induced by the tadpole corrections: $\left\langle\psi_{l}\right\rangle=\alpha+\left\langle\tilde{\psi}_{l}\right\rangle \neq \alpha$. An alternative procedure is thus to fix $\alpha$ by the condition that tadpole 1-particle irreducible diagrams vanish. This condition guarantees that $\left\langle\psi_{l}\right\rangle=\alpha$, since the vanishing of 1-particle irreducible diagrams implies also the vanishing of all reducible ones contributing to $\left\langle\psi_{l}\right\rangle$. The equation for $\alpha$ is then calculated perturbatively as a loopexpansion without tadpole insertions:

$$
F^{(0)}(\alpha)+F^{(1)}(\alpha)+F^{(2)}(\alpha)+\ldots=0
$$

where $F^{(0)}(\alpha)=2 \alpha\left(\mu-v \alpha^{2}\right)$ and $F^{(n)}(\alpha)$ is the sum of all one-leg n-loop diagrams (cfr. (B1) and (B2) for the one-loop case). Substituting the loop expansion of $\alpha\left(\alpha=\alpha^{(0)}+\alpha^{(1)}+\ldots\right)$ and solving for $\alpha$ order by order, one obtains $\alpha^{(0)}=\mu / v, \alpha^{(1)}=$ $-F^{(1)}\left(\alpha^{(0)}\right) /\left(\partial F^{(0)}(\alpha) / \partial \alpha\right)_{\alpha=\alpha^{(0)}}, \ldots$. This procedure defines a much simpler diagrammatics, since no tadpole diagrams are present, their contribution appearing as a shift of $\alpha$. The price to pay is that one has to calculate all diagrams for arbitrary $\alpha$ both in the 3-leg couplings and propagators. This implies that the propagators would have a mass (gap), but since all quantities have to be expanded around their mean-field values one recovers massless propagators in all expressions. ${ }^{19}$ This fixes the procedure in a precise way.

In our case an important simplification occurs. As we are interested in the IR behavior of the exact propagators, finite corrections are not important. Since the tadpole diagrams are all finite in the infrared, we will not calculate the finite corrections to $\alpha$ accordingly. In the following, $\alpha$ can be then thought as fixed and to coincide with $\psi_{l o}$. At the same time, the propagators are massless and no tadpole diagrams will be considered. In practice, this means that finite corrections to the condensate are not important down to $d=1$. At that point the condensate vanishes due to IR divergences. We will see that precisely at $d=1$ our approach breaks down since the the longitudinal and transverse fluctuations becomes equally singular.

As far as the chemical potential entering the action (2.16) is concerned, it is possible to leave its value unspecified in the following calculation since the interacting boson system with a fixed chemical potential is well defined. ${ }^{20}$

PT simplifies by introducing the Legendre transform of $F$ with respect to the sources $\lambda$ :

$$
\Gamma\left[\psi_{i o}, A_{\nu}\right]=\int d x \lambda_{i}(x) \psi_{o i}(x)-F\left[\lambda_{i}\left[\psi_{o i}\right], A_{\nu}\right]
$$

where

$$
\delta \Gamma / \delta \psi_{i o}(x)=\lambda_{i}(x)
$$

and

$$
\delta \Gamma / \delta A_{\nu}(x)=-\delta F / \delta A_{\nu}(x)
$$

Similarly, the connected correlation functions and the vertex functions are respectively given by: 


$$
\begin{gathered}
\frac{\delta^{(n+m)} F}{\delta \lambda_{i_{1}}\left(x_{1}\right) \ldots \delta \lambda_{i_{n}}\left(x_{n}\right) \delta A_{\nu_{1}}\left(y_{1}\right) \ldots \delta A_{\nu_{m}}\left(y_{m}\right)}= \\
-\mathcal{G}_{i_{1} \ldots i_{n} ; \nu_{1} \ldots \nu_{m}}\left(x_{1}, \ldots, x_{n} ; y_{1}, \ldots, y_{m}\right), \\
\frac{\delta^{(n+m)} \Gamma}{\delta \psi_{i_{1} o}\left(x_{1}\right) \ldots \delta \psi_{i_{n} o}\left(x_{n}\right) \delta A_{\nu_{1}}\left(y_{1}\right) \ldots \delta A_{\nu_{m}}\left(y_{m}\right)}= \\
\Gamma_{i_{1} \ldots i_{n} ; \nu_{1} \ldots \nu_{m}}\left(x_{1}, \ldots, x_{n} ; y_{1}, \ldots, y_{m}\right) .
\end{gathered}
$$

Here, the derivatives with respect to $\lambda_{i}$ give the connected and amputated (1-particle irreducible) correlation functions, for $F$ and $\Gamma$, respectively The derivatives with respect to $A_{\nu}$ generate the current and density response functions (or their amputated counterparts).

The single-particle Green's functions analogous to Eq. (2.9) can be obtained from the quadratic part of the action (2.16), which reads in terms of the independent fields:

$$
S_{Q}=\sum_{k, i, j} \tilde{\psi}_{i}^{*}(k) M_{i j}(k) \tilde{\psi}_{j}(k)
$$

with

$$
M(k)=\left(\begin{array}{cc}
\mu-3 v \alpha^{2}-\mathbf{k}^{2} & -\omega_{s} \\
\omega_{s} & \mu-v \alpha^{2}-\mathbf{k}^{2}
\end{array}\right) .
$$

With the aid of the condition (2.8), one then obtains:

$$
\mathcal{G}(k)=\frac{1}{2}\left(M^{-1}\right)^{t}=-\frac{1}{2} \frac{1}{\omega_{s}^{2}+E_{\mathbf{k}}^{2}}\left(\begin{array}{cc}
\mathbf{k}^{2} & \omega_{s} \\
-\omega_{s} & 2 \mu+\mathbf{k}^{2}
\end{array}\right)
$$

which for small $\mathbf{k}$ and $\omega_{s}$ behaves like

$$
\left\{\begin{array}{l}
\mathcal{G}_{t t}(k) \sim-c_{o}^{2} /\left(\omega_{s}^{2}+c_{o}^{2} \mathbf{k}^{2}\right) \\
\mathcal{G}_{l l}(k) \sim-\omega_{s} /\left(\omega_{s}^{2}+c_{o}^{2} \mathbf{k}^{2}\right) \\
\mathcal{G}_{l l}(k) \sim-\mathbf{k}^{2} /\left(\omega_{s}^{2}+c_{o}^{2} \mathbf{k}^{2}\right) .
\end{array}\right.
$$

In the present case the low-energy region is dominated by the Goldstone (sound) mode. The IR limit of the correlation functions is thus naturally defined by taking frequency and wave vector to vanish while maintaining their ratio constant. For example, one can take $\lim _{\gamma \rightarrow 0} \mathcal{G}_{i j}\left(\gamma \omega_{s}, \gamma \mathbf{k}\right)$. In this way, the three propagators (2.27) have different IR behavior, with the strongest singularity residing in the transverse propagator $\mathcal{G}_{t t}$. This situation has to be contrasted with the standard $\psi$ representation, where the Bogoliubov propagators (2.9) share instead the same IR behavior. The choice of $\left(\psi_{l}, \psi_{t}\right)$ in the place of $\left(\psi, \psi^{*}\right)$ will thus turn out to be crucial to select the interaction terms on the basis of their relevance. Furthermore, this choice will be important from a physical point of view since an anomalous dimension will appear in the longitudinal component $\mathcal{G}_{l l}$ only.

To treat these singularities, it is important to classify the coupling terms in the action (2.16) according to their relevance in generating IR divergences. A standard way to perform this task is to define scaled fields and frequencies, so that the infrared dimensions of the propagators in (2.27) coincide with their engineering dimensions. In our case the scaling is defined as follows:

$$
\left\{\begin{array}{l}
\bar{\psi}_{l}\left(k_{0}, \mathbf{k}\right)=c_{o} \tilde{\psi}_{l}\left(c_{o} k_{0}, \mathbf{k}\right) \\
\bar{\psi}_{t}\left(k_{0}, \mathbf{k}\right)=\tilde{\psi}_{t}\left(c_{o} k_{0}, \mathbf{k}\right)
\end{array}\right.
$$

with the short-hand notation $k_{0}=i \omega_{s} / c_{o}$ (from now on $\left.k=\left(k_{0}, \mathbf{k}\right)\right)$. Since the rescaled fields have dimensions $\left[\bar{\psi}_{l}(k)\right]=\left[\tilde{\psi}_{l}(k)\right]+1=0$ and $\left[\bar{\psi}_{t}(k)\right]=\left[\tilde{\psi}_{t}(k)\right]=-1$ (we have defined the scale of the momentum as the unit, $[k]=1)$, this leads to the desired dimensions for the propagators of the rescaled fields:

$$
\left[\overline{\mathcal{G}}_{t t}(k)\right]=-2, \quad\left[\overline{\mathcal{G}}_{l t}(k)\right]=-1, \quad\left[\overline{\mathcal{G}}_{l l}(k)\right]=0 .
$$

Since different diagrams contributing to the same quantity must have the same dimension, couplings of higher dimension must appear together with most divergent Green's functions. Thus the engineering dimensions of the couplings for the new fields can be used to classify their relevance. Equation (2.28) implies that, in real space, the fields are rescaled as

$$
\left\{\begin{array}{l}
\bar{\psi}_{l}\left(x_{0}, \mathbf{x}\right)=c_{o}^{1 / 2} \bar{\psi}_{l}\left(x_{0} / c_{o}, \mathbf{x}\right) \\
\bar{\psi}_{t}\left(x_{0}, \mathbf{x}\right)=c_{o}^{-1 / 2} \bar{\psi}_{t}\left(x_{0} / c_{o}, \mathbf{x}\right),
\end{array}\right.
$$

which follows from a redefinition of the Fourier transform:

$$
\bar{\psi}_{i}\left(k_{0}, \mathbf{k}\right)=\frac{1}{\sqrt{\beta c_{o} \Omega}} \int_{0}^{\beta c_{o}} d x_{0} \int d^{d} \mathbf{x} e^{-i k x} \bar{\psi}_{i}(x) .
$$

The dimension of the fields are $\left[\psi_{l}(x)\right]=(d+1) / 2$ and $\left[\psi_{t}(x)\right]=(d-1) / 2$. From the above considerations, the dimension of a generic coupling $v_{n_{l} n_{t}}$ associated with the monomial $\bar{\psi}_{l}^{n_{l}}(x) \bar{\psi}_{t}^{n_{t}}(x)$ is given by

$$
\left[v_{n_{l}, n_{t}}\right]=-n_{l} \frac{d+1}{2}-n_{t} \frac{d-1}{2}+d+1 .
$$

For later convenience, we write explicitly the dimensions of the couplings up to four legs:

$$
\begin{gathered}
\left\{\begin{array}{l}
{\left[v_{l}\right]=2-\epsilon / 2} \\
{\left[v_{t}\right]=3-\epsilon / 2}
\end{array},\left\{\begin{array}{l}
{\left[v_{l l}\right]=0} \\
{\left[v_{l t}\right]=1} \\
{\left[v_{t t}\right]=2}
\end{array}\right.\right. \\
\left\{\begin{array}{l}
{\left[v_{l l l}\right]=-2+\frac{\epsilon}{2}} \\
{\left[v_{l l t}\right]=-1+\frac{\epsilon}{2}} \\
{\left[v_{l t t}\right]=\frac{\epsilon}{2}} \\
{\left[v_{t t t}\right]=1+\frac{\epsilon}{2}}
\end{array},\left\{\begin{array}{l}
{\left[v_{l l l l}\right]=-4+\epsilon} \\
{\left[v_{l l l t}\right]=-3+\epsilon} \\
{\left[v_{l l t t}\right]=-2+\epsilon} \\
{\left[v_{l t t t}\right]=-1+\epsilon} \\
{\left[v_{t t t t}\right]=\epsilon}
\end{array}\right.\right.
\end{gathered}
$$

where $v_{l}=v_{10}, v_{l l}=v_{20}, v_{l t t}=v_{12}$, etc., and $\epsilon=3-d$ parameterizes the distance from the critical dimension $d_{c}=3$ at which logarithmic divergences appear.

Couplings with more than four legs have not been considered since they are irrelevant at $d=d_{c}$, i.e., they have negative dimensions. The vertex functions have 
the same dimensions of the relative coupling constants. This implies, for instance, that the tadpole equation is non-divergent in the infrared, as anticipated, since $\left[v_{l}\right]=\left[\Gamma_{l}\right]=2-\epsilon / 2>0$

To make PT calculations (as well as the RG treatment) more convenient, we generalize the original action (2.16) in the broken-symmetry phase as follows. Besides the terms already present in (2.16), we introduce additional running couplings which are relevant or marginal at $d_{c}$ (while all irrelevant couplings were already present in the original action). The generalized action thus acquires the form: ${ }^{21}$

$$
\begin{aligned}
& S\left[\bar{\psi}_{i}\right]=\beta \Omega v_{0}-(\beta \Omega)^{1 / 2} v_{l} \bar{\psi}_{l}(0)-(\beta \Omega)^{1 / 2} v_{t} \bar{\psi}_{t}(0) \\
& -\frac{1}{2 !} \sum_{k}\left\{\left[v_{l l}+z_{l l} \mathbf{k}^{2}\right] \bar{\psi}_{l}(-k) \bar{\psi}_{l}(k)+2\left[v_{l t}+w_{l t} \omega_{s}\right] \bar{\psi}_{l}(-k) \bar{\psi}_{t}(k)+\left[v_{t t}+u_{t t} \omega_{s}^{2}+z_{t t} \mathbf{k}^{2}\right] \bar{\psi}_{t}(-k) \bar{\psi}_{t}(k)\right\} \\
& -\frac{(\beta \Omega)^{-1 / 2}}{3 !} \sum_{k_{1}+k_{2}+k_{3}=0}\left\{v_{t t t} \bar{\psi}_{t}\left(k_{1}\right) \bar{\psi}_{t}\left(k_{2}\right) \bar{\psi}_{t}\left(k_{3}\right)+3 v_{l t t} \bar{\psi}_{l}\left(k_{1}\right) \bar{\psi}_{t}\left(k_{2}\right) \bar{\psi}_{t}\left(k_{3}\right)+v_{l l l} \bar{\psi}_{l}\left(k_{1}\right) \bar{\psi}_{l}\left(k_{2}\right) \bar{\psi}_{l}\left(k_{3}\right)\right\} \\
& -\frac{(\beta \Omega)^{-1}}{4 !} \sum_{\sum_{i} k_{i}=0}\left\{v_{t t t t} \bar{\psi}_{t}\left(k_{1}\right) \bar{\psi}_{t}\left(k_{2}\right) \bar{\psi}_{t}\left(k_{3}\right) \bar{\psi}_{t}\left(k_{4}\right)+6 v_{l l t t} \bar{\psi}_{l}\left(k_{1}\right) \bar{\psi}_{l}\left(k_{2}\right) \bar{\psi}_{t}\left(k_{3}\right) \bar{\psi}_{t}\left(k_{4}\right)+v_{l l l l} \bar{\psi}_{l}\left(k_{1}\right) \bar{\psi}_{l}\left(k_{2}\right) \bar{\psi}_{l}\left(k_{3}\right) \bar{\psi}_{l}\left(k_{4}\right)\right\}
\end{aligned}
$$

The reason to use these new couplings in the place of the original ones is related to the fact that they appears as coefficients of the monomials of fields, and thus have a simple bare scaling behavior. It is also clear that there is a definite relation among the new set of couplings and the original ones, that can be inferred by direct comparison of (2.16) and (2.34). Since the new set of couplings is larger, it is also clear that they will not be independent when related to the original parameters. In Section III A these connections will be discussed and related to the gauge invariance of the original theory.

As far as the marginal couplings $\left(w_{l t}, u_{t t}, z_{t t}\right)$ are concerned, we recall that in the original action (2.16) they had, respectively, the values $(2,0,2)$ regardless of the original parameters $(v, \mu, \alpha)$. We follow here a standard procedure of the field-theoretical RG treatment and let the coefficients of the marginal terms proportional to $\omega, \omega^{2}$, and $\mathbf{k}^{2}$ assume arbitrary values in order to renormalize the theory. In principle, an alternative procedure would be to introducing two wave-function-like renormalization parameters and one dynamical renormalization parameter which, in turn, allows one to keep the matrix of the propagators unchanged. It will turn out, however, that the renormalization of these parameters, although nontrivial, will not affect the scaling form of the matrix of the renormalized propagators, since these parameters will appear there only in some specific combinations that are independent of the RG flow. It will, in fact, turn out that the anomalous behavior of the longitudinal propagator depends only on the renormalization of the coupling $v_{l l}$; at the same time, the dynamical index will stay finite at $z=1$, consistently with the Bogoliubov sound mode.

Quite generally, the matrix entering the quadratic part of the action (2.34) is defined as in (2.24) and is given by:

$$
M=-\left(\begin{array}{cc}
v_{l l}+z_{l l} \mathbf{k}^{2}, & v_{l t}+w_{l t} \omega_{s} \\
v_{t l}-w_{l t} \omega_{s}, & v_{t t}+u_{t t} \omega_{s}^{2}+z_{t t} \mathbf{k}^{2}
\end{array}\right)
$$

in the place of Eq. (2.25). The corresponding singleparticle propagator becomes:

$$
\begin{aligned}
\overline{\mathcal{G}}(k) & =-\left(\begin{array}{cc}
v_{t t}+u_{t t} \omega_{s}^{2}+z_{t t} \mathbf{k}^{2}, & -v_{t l}+w_{l t} \omega_{s} \\
-v_{l t}-w_{l t} \omega_{s}, & v_{l l}+z_{l l} \mathbf{k}^{2}
\end{array}\right) \frac{1}{D(k)} \\
& \equiv\left(\begin{array}{cc}
\overline{\mathcal{G}}_{l l}(k) & \overline{\mathcal{G}}_{l t}(k) \\
\overline{\mathcal{G}}_{t l}(k) & \overline{\mathcal{G}}_{t t}(k)
\end{array}\right)
\end{aligned}
$$

with

$$
\begin{aligned}
D(k)= & v_{l l} v_{t t}-v_{l t}^{2}+\left(u_{t t} v_{l l}+w_{l t}^{2}\right) \omega_{s}^{2} \\
& +\left(v_{l l} z_{t t}+v_{t t} z_{l l}+z_{l l} z_{t t} \mathbf{k}^{2}\right) \mathbf{k}^{2}
\end{aligned}
$$

in the place of Eq. (2.26). For later convenience, the components of the propagator (2.36) are depicted schematically as follows:

$$
\mathcal{G}_{l l}(k)=---, \mathcal{G}_{l t}(k)=---, \mathcal{G}_{t t}(k)=-
$$

The remaining terms of the action beyond the quadratic part are considered as perturbation. The interaction terms retained in the $\mathrm{RG}$ treatment are:

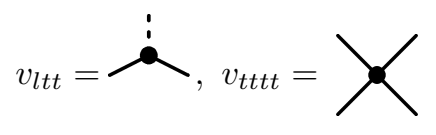

PT treatment then proceeds now along the usual lines.

Although the generalized action (2.34) contains four marginal couplings $\left(v_{l l}, w_{l t}, u_{t t}, z_{t t}\right)$ and seven relevant couplings $\left(v_{l}, v_{t}, v_{l t}, v_{t t}, v_{l t t}, v_{t t t}, v_{t t t t}\right)$, it will be shown in the next Section via the use of WI that these couplings are actually not independent from each other. 


\section{GAUGE INVARIANCE AND WARD IDENTITIES}

The original action (2.1) satisfies local-gauge invariance, in the sense that it is unaffected by the following transformation:

$$
\begin{cases}\psi(x) \rightarrow \psi(x) e^{i \chi(x)} & \psi(x)^{*} \rightarrow \psi(x)^{*} e^{-i \chi(x)} \\ \lambda(x) \rightarrow \lambda(x) e^{-i \chi(x)} & \lambda(x)^{*} \rightarrow \lambda(x)^{*} e^{i \chi(x)} \\ \mu(x) \rightarrow \mu(x)-i \partial_{\tau} \chi(x) & \mathbf{A}(x) \rightarrow \mathbf{A}(x)-\nabla \chi(x) .\end{cases}
$$

This invariance provides definite connections (in the form of WI) among the vertex functions (2.23)..$^{-7}$ These identities have been exploited by Gavoret and Nozières to establish connections between vertex functions and thermodynamic derivatives for vanishing wave vector and frequency. In this Section, we shall make use of the WI in two additional ways. On the one hand, we shall constraint the various couplings of the generalized action (2.34) by showing that they are not independent from each other when the generalized action is required to be gauge invariant. On the other hand, we will exploit the WI to connect the IR singular parts of the vertex functions in PT and to implement their cancellation to all orders in the response functions.

To be consistent with our notation, we first give a short derivation of the set of WI relevant to our purposes. In terms of the longitudinal and transverse components $\left(\psi_{l}\right.$, $\left.\psi_{t}\right)$, the original action (2.1) has the following invariance:

$$
S\left[R_{i j} \psi_{j}, R_{i j} \lambda_{j}, A_{\nu}-\partial_{\nu} \chi\right]=S\left[\psi_{i}, \lambda_{i}, A_{\nu}\right]
$$

where $i$ and $j$ take the values $(l, t)$ and a summation over repeated indices is implied. In Eq. (3.2) we have introduced the notation $\partial_{\nu}=\left(i \partial_{\tau}, \nabla\right)$ and $\mathbf{R}$ is the rotation matrix

$$
\mathbf{R}=\left(\begin{array}{cc}
\cos \chi(x) & -\sin \chi(x) \\
\sin \chi(x) & \cos \chi(x)
\end{array}\right)
$$

$\chi(x)$ being an arbitrary real function. The above invariance leads, in turn, to the following invariance of the functional $\Gamma$ :

$$
\Gamma\left[A_{\nu}-\partial_{\nu} \chi(x), R_{i j} \psi_{j o}\right]=\Gamma\left[A_{\nu}, \psi_{i o}\right]
$$

The desired connections among the vertex functions are obtained at this point by differentiating Eq. (3.4) with respect to $\chi$. One obtains:

$$
\Gamma_{i}(x) \sigma_{i j} \psi_{j o}(x)+\partial_{\nu} \Gamma_{; \nu}(x)=0
$$

where $\sigma$ is the infinitesimal generator of rotations

$$
\sigma=\left(\begin{array}{cc}
0 & -1 \\
1 & 0
\end{array}\right)
$$

and $\chi$ has been set to zero eventually. Successive differentiations with respect to $\psi_{i o}$ give in addition:

$$
\begin{aligned}
& \Gamma_{i m}\left(x_{1}, x_{2}\right) \sigma_{i j} \psi_{j o}\left(x_{1}\right)+\Gamma_{i}\left(x_{1}\right) \sigma_{i m} \delta\left(x_{1}-x_{2}\right)+\partial_{\nu}^{x_{1}} \Gamma_{m ; \nu}\left(x_{2} ; x_{1}\right)=0 \\
& \Gamma_{i m n}\left(x_{1}, x_{2}, x_{3}\right) \sigma_{i j} \psi_{j o}\left(x_{1}\right)+\Gamma_{i m}\left(x_{1}, x_{2}\right) \sigma_{i n} \delta\left(x_{1}-x_{3}\right)+\Gamma_{i n}\left(x_{1}, x_{3}\right) \sigma_{i m} \delta\left(x_{1}-x_{2}\right) \\
& +\partial_{\nu}^{x_{1}} \Gamma_{m n ; \nu}\left(x_{2}, x_{3} ; x_{1}\right)=0
\end{aligned}
$$

and

$$
\begin{aligned}
& \Gamma_{i m n r}\left(x_{1}, x_{2}, x_{3}, x_{4}\right) \sigma_{i j} \psi_{j o}\left(x_{1}\right)+\Gamma_{i m n}\left(x_{1}, x_{2}, x_{3}\right) \sigma_{i r} \delta\left(x_{1}-x_{4}\right)+\Gamma_{i m r}\left(x_{1}, x_{2}, x_{4}\right) \sigma_{i n} \delta\left(x_{1}-x_{3}\right) \\
& +\Gamma_{i n r}\left(x_{1}, x_{3}, x_{4}\right) \sigma_{i m} \delta\left(x_{1}-x_{2}\right)+\partial_{\nu}^{x_{1}} \Gamma_{m n r ; \nu}\left(x_{2}, x_{3}, x_{4} ; x_{1}\right)=0 .
\end{aligned}
$$

The WI (3.5)-(3.9) are sufficient for the RG treatment to be discussed in Section IV.

For homogeneous external sources, in terms of Fourier transforms (for instance, $\Gamma_{i}(x)=\frac{1}{\beta \Omega} \sum_{q} e^{i q x} \Gamma_{i}(q)$ with the short-hand notation $\left.q x=\omega_{s} \tau+\mathbf{k} \cdot \mathbf{r}\right)$ the WI (3.5)(3.9) simplify considerably. In particular, the sets of WI which will be explicitly used in the following for vanishing transverse source (i.e., $\psi_{\text {to }}=0$ ) can be grouped as follows:

$$
\begin{gathered}
\Gamma_{t} \psi_{l o}=0 \\
\Gamma_{t l}(k) \psi_{l o}+\Gamma_{t}-i k_{\nu} \Gamma_{l ; \nu}(-k)=0 \\
\Gamma_{t t}(k) \psi_{l o}-\Gamma_{l}-i k_{\nu} \Gamma_{t ; \nu}(-k)=0
\end{gathered}
$$

from (3.5) and (3.7), which encompass the HugenholtzPines $^{2}$ identity for $k=0$;

$$
\begin{aligned}
& \Gamma_{t t l}\left(k_{1}, k_{2}\right) \psi_{l o}+\Gamma_{t t}\left(-k_{2}\right)-\Gamma_{l l}\left(k_{1}+k_{2}\right) \\
& -i\left(k_{1}\right)_{\nu} \Gamma_{t l ; \nu}\left(k_{2},-k_{1}-k_{2}\right)=0 \\
& \Gamma_{t t t}\left(k_{1}, k_{2}\right) \psi_{l o}-\Gamma_{l t}\left(-k_{2}\right)-\Gamma_{l t}\left(k_{1}+k_{2}\right) \\
& -i\left(k_{1}\right)_{\nu} \Gamma_{t t ; \nu}\left(k_{2},-k_{1}-k_{2}\right)=0
\end{aligned}
$$

which follow from (3.8) and are the standard WI associated with the continuity equation modified by the presence of the 3-legs vertices; and from (3.9) the four legs ones

$$
\Gamma_{t t t t}\left(k_{1}, k_{2}, k_{3}\right) \psi_{l o}-\Gamma_{l t t}\left(-k_{2}-k_{3}, k_{2}\right)
$$




$$
\begin{aligned}
& -\Gamma_{l t t}\left(k_{1}+k_{3}, k_{2}\right)-\Gamma_{l t t}\left(k_{1}+k_{2}, k_{3}\right) \\
& -i\left(k_{1}\right)_{\nu} \Gamma_{t t t ; \nu}\left(k_{2}, k_{3},-k_{1}-k_{2}-k_{3}\right)=0 .
\end{aligned}
$$

Here, $k_{\nu}=\left(i \omega_{s}, \mathbf{k}\right)$ with $\nu=(0, \ldots, d)$ and the conservation of momentum has been used to eliminate the dependence on the last momentum of each vertex function, i.e., $\Gamma_{i}(k)=\Gamma_{i}(k=0) \equiv \Gamma_{i}, \Gamma_{i j}\left(k_{1}, k_{2}\right) \equiv \Gamma_{i j}\left(k_{1}\right)$, and so on. It is thus understood that the vertex functions are free from the overall momentum conserving delta function. We include the $i$ in the definition of $k_{0}$ only in the scalar product entering the WI to simplify the notation; everywhere else $k_{0}=\omega_{s}$. The rescaling (2.28) has not been considered explicitly in the present Section, but it can be readily introduced whenever necessary.

We recall that the WI (3.10)-(3.15) have been obtained using the invariance properties of the original action (2.16). For arbitrary values of the couplings, the generalized action (2.34) will not be invariant under the transformation (3.2). Thus the requirement of fulfilling (3.2) will provide constraints on the couplings appearing in (2.34). It is also clear that this requirement by itself is not sufficient to reduce the number of independent couplings to the original ones. It is, in fact, possible to add to the original action (2.1) terms of the type $v_{n}|\psi|^{2 n}$ still preserving gauge invariance. To identify (2.16) exactly with (2.34) one should specify, for instance, that no 5-leg couplings are present. With this additional condition, it can be shown that the independent couplings left are only four (for instance, $v_{l}, v_{l l}, \psi_{l o}$, and $\psi_{t o}$ ) corresponding to $\mu, v, \lambda_{l}$, and $\lambda_{t}$ of the original action (2.1).

In Appendix A, we discuss in more details the formal definition of gauge invariance in terms of the couplings introduced in this Section. There we show how to enforce the invariance (3.1) directly on the action. In an equivalent way here we proceed by assuming that the new action (2.34) shares the same invariance properties of (2.1). Then Eq. (3.4) holds and the above WI follow. We thus enforce the vertex functions associated with the new action to satisfy these WI and obtain relationships among the bare couplings of the action (2.16).

To be more precise, we can calculate all vertex functions by a loop expansion, since the WI are preserved order by order. We can use this method to: (i) Constraint the bare couplings using the lowest order 0-loops expressions; (ii) Connect the divergent contributions to different renormalized running couplings at any loop and thus find relations among themselves.

\section{A. Global Ward identities: $k=0$}

We are now in a position to discuss the vanishing of the relevant bare couplings $v_{l}, v_{t}, v_{l t}, v_{t t}$, and $v_{t t t}$ present in (2.34) at any dimension.

At the lowest order in the loop expansion, Eqs. (3.10)(3.12) and (3.14) can be evaluated at vanishing $k$ and the vertex functions expressed in terms of the bare couplings. This procedure provides definite relationships among the relevant bare couplings. Specifically, we obtain $v_{t}=0$ from Eq. (3.10) for non vanishing condensate density $\psi_{l o}$; $v_{l t} \psi_{l o}+v_{t}=0$ from Eq. (3.11); $v_{t t} \psi_{l o}-v_{l}=0$ from Eq. (3.12); and $v_{t t t} \psi_{l o}-2 v_{l t}=0$ from Eq. (3.14). This gives:

$$
v_{t}=0, \quad v_{l t}=0, \quad v_{t t t}=0,
$$

and

$$
v_{l}=\psi_{l o} v_{t t}
$$

for vanishing $\psi_{t o}$. To determine $v_{l}$, we consider in addition the "equation of state" (2.20) (with $i=l$ ) for an isolated system with $\lambda_{i}=0$. This is the counterpart of the condensate equation (2.18) in the new variables and fixes the bare coupling $v_{l}=0$. Actually, this is not true at higher orders, since the shift of the condensate will induce finite corrections to $v_{l}$ that must be fixed in order to let tadpole diagrams vanish. Since we are not interested in finite corrections, we can set the bare and renormalized couplings $v_{l}=0$, and thus $v_{t t}=0$. The vanishing of $v_{t t}$ implements the Hugenholtz-Pines identity, leading to gapless propagators.

We pass now to discuss the connections among the marginal couplings. Let's consider the bare marginal couplings first. The identities (3.13) and (3.15) can be interpreted at the lowest-order of the loop expansion as follows:

$$
\begin{array}{r}
v_{l t t} \psi_{l o}-v_{l l}=0 \\
v_{t t t t} \psi_{l o}-3 v_{l t t}=0
\end{array}
$$

where all $k$ have been taken to vanish. The marginal bare couplings thus eventually reduce to four independent ones $v_{l l}, w_{l t}, u_{t t}$, and $z_{t t}$.

It is clear that the relations (3.16), (3.17), (3.18), and (3.19) must be enforced on the bare running couplings to guarantee the gauge invariance of the theory. A field theoretic RG treatment is now possible only if we neglect all irrelevant running couplings. Although this spoils gauge invariance, it does not matter for what concerns the leading order-singularities. We can verify that gauge invariance is lost if we set all irrelevant running couplings to zero, since in this case the WI (at the lowest-order in loops) are no longer compatible with finite values of the marginal running couplings. In other words, the use of a perturbation theory for the $\Gamma$ 's with only the marginal running couplings will violate the WI (as shown explicitly in Appendix B), but the leading diverging contribution to each $\Gamma$ function is fully retained by this approximation. This implies that the finite part of the WI can be violated, but the divergent contributions coming from different $\Gamma$ functions will correctly simplify in the WI. Thus the WI hold for the renormalization of each vertex function, since these are introduced exactly to subtract out the divergent parts. For this reason the WI (3.18)(3.19) will hold for the renormalized running couplings too. 


\section{B. Local Ward identities: small $k$}

There remains to consider the connections between the couplings $\left(w_{l t}, u_{t t}, z_{t t}\right)$, associated with the wave vector and frequency dependence in the action (2.34), with the thermodynamic derivatives in the limit of vanishing $\mathbf{k}$ and $\omega$. To this end, we consider the vertex functions $\Gamma_{l l}$, $\Gamma_{l t}$, and $\Gamma_{t t}$ associated with the couplings of interest. The long-wavelength limit of these vertex functions has been obtained long ago. ${ }^{4-6}$ Here, we will rederive them in a more compact form taking into account the presence of IR divergences. Important information on the scaling of the running coupling will follow from these connections.

We begin by establishing their symmetry properties. From previous considerations $\Gamma_{l t}(k=0)=0$ and $\Gamma_{t t}(k=$ $0)=0$. Using the symmetry properties of the action (2.34) under space and time reversal and under exchange of the two components $l$ and $t$ of the bosonic field, one can show that $\Gamma_{l t}(k)$ is odd under the replacement $\omega_{s} \rightarrow-\omega_{s}$ while $\Gamma_{t t}$ is even. We then take the zero-temperature limit (whereby $\omega_{s}$ becomes a continuous variable, denoted by $\omega$ from now on) and consider the WI (3.11) and (3.12).

It is convenient to cast the vertex functions in a form that makes their symmetry properties manifest. For instance, from $\Gamma_{l t}(\omega, \mathbf{k})=-\Gamma_{l t}(-\omega, \mathbf{k})$ we write

$$
\Gamma_{l t}(\omega, \mathbf{k})=\omega \mathcal{P}_{l t}\left(\omega, \mathbf{k}^{2}\right)
$$

where $\mathcal{P}_{l t}\left(\omega, \mathbf{k}^{2}\right)$ is an even function of $\omega_{s}$. Similarly, we can write

$$
\Gamma_{l ; 0}(k)=\mathcal{P}_{l ; 0}\left(\omega, \mathbf{k}^{2}\right)
$$

and

$$
\Gamma_{l ; n}(k)=\omega i k_{n} \mathcal{P}_{l ; v}\left(\omega, \mathbf{k}^{2}\right)
$$

$(n=1, \ldots, d)$ where $\mathcal{P}_{l ; 0}(k)$ and $\mathcal{P}_{l ; v}(k)$ are even functions of $\omega$ and depends on $\mathbf{k}$ only through $\mathbf{k}^{2}$ (note that the suffix $v$ is here a reminder that we are considering a vector function). In this way, the WI (3.11) becomes:

$$
\psi_{l o} \mathcal{P}_{l t}(k)+\mathcal{P}_{l ; 0}(k)+\mathbf{k}^{2} \mathcal{P}_{l ; v}(k)=0
$$

where Eq. (3.10) has been taken into account.

To the purpose of understanding the behavior of the singularities when we let $\omega$ or $\mathbf{k}$ vanish indepentently, we discuss the form of the divergences. At the critical dimension $\left(d_{c}=3\right)$, a generic vertex function $\Gamma(k)$ of a single external variable in the limit $k \rightarrow 0$ can be written formally as

$$
\begin{aligned}
\Gamma(k)= & \left(k^{2}\right)^{D / 2}\left[\sum_{m=1}^{\infty} A_{m} \ln ^{m}\left(\mathbf{k}^{2}+\frac{\omega^{2}}{c_{o}^{2}}\right)+B\right] \\
& +C
\end{aligned}
$$

where $D$ stands for the IR dimension of $\Gamma(k)$ and $A_{m}$, $B$, and $C$ are constants. [In general, instead of the term
$\left(k^{2}\right)^{D / 2}$ one can use $\left(\omega^{2}\right)^{D / 2},\left(\mathbf{k}^{2}\right)^{D / 2}$, or any linear combination of the two.] In this expression, we are not considering the terms arising from the internally diverging diagrams, since the divergent contributions due to these diagrams are eliminated by the renormalization at lower order of the other $\Gamma$ functions. In this scheme, the divergences left are all due to the primitively divergent diagrams of each vertex function. These divergences must cancel out in the WI, so exact relations result among the primitively divergent contributions to each $\Gamma$ function. The form (3.24) implies also that the $k \rightarrow 0$ limit of the divergent $\log$ series does not depend on which limit is taken first, $\omega \rightarrow 0$ or $\mathbf{k} \rightarrow 0$. This is a consequence of the fact that all singular dependence comes through the single argument of the log terms. This fact can be verified order by order in loops [see Appendix B]. Of course, the order in which the limits are taken affects the final value of the $\Gamma$ functions that have overall $\omega$ and $\mathbf{k}$ terms. The $\Gamma$ functions that have $D=0$ will be totally symmetric, and the information gained for one of the two variables set to zero applies also for $k \rightarrow 0$. Knowing the degree of divergence of the vertex functions and the explicit combinations of $\omega$ and $\mathbf{k}$ appearing in each expression, one can establish relations between the vertex functions in presence of IR divergences.

In order to proceed further we need the IR dimension (i.e., the engineering dimension after the rescaling (2.28)) of the composite vertex parts. One should consider, however, that IR dimension is correctly defined for monomials of fields. For instance, we can readily obtain that the dimension of $\Gamma_{l ; l^{2}}$ is $2-\epsilon / 2$, where by $l^{2}$ we mean the field $\psi_{l}^{2}\left(\mathcal{G}_{; l^{2}}(x) \equiv-\left\langle\psi_{l}^{2}(x)\right\rangle\right)$. But the dimension of a physical composite fields will mix different monomials and thus different IR behavior: $\Gamma_{l ; 0}=\Gamma_{l ; l^{2}}+\Gamma_{l ; t^{2}}$. As we are concerned with divergences, we identify the IR dimension of a composite field with the most divergent dimension (in the above example with $\left[\Gamma_{l ; t^{2}}\right]=-\epsilon / 2$ we define $\left.\left[\Gamma_{l ; 0}\right]=-\epsilon / 2\right)$.

We report the IR dimension of other relevant $\Gamma$ functions for further reference:

$$
\left\{\begin{array} { l } 
{ [ \overline { \Gamma } _ { l ; j } ] = 2 - \epsilon } \\
{ \overline { \Gamma } _ { t ; 0 } ] = 1 - \epsilon } \\
{ [ \overline { \Gamma } _ { t ; j } ] = 2 - \epsilon , }
\end{array} \quad \left\{\begin{array}{l}
{\left[\bar{\Gamma}_{i 00}\right]=-\epsilon} \\
{\left[\bar{\Gamma}_{; i 0}=2-\epsilon\right.} \\
{\left[\bar{\Gamma}_{; i j}\right]=4-\epsilon}
\end{array}\right.\right.
$$

These identities are obtained by performing the Fourier transform of the Green's function, taking into account that square of fields depend on a single variable. ${ }^{14}$

We pass now to discuss the WI (3.23). Since the functions entering (3.23) have vanishing IR dimensions [cf. Eqs. (3.25) and (2.33) taking also into accont that the functions $\mathcal{P}$ differ from the respective $\Gamma$ by factors $\omega$ or $\mathbf{k}$ ], the last term on the left-hand side of (3.24) vanishes as $\mathbf{k}^{2} \ln ^{m}\left(\mathbf{k}^{2}+\omega^{2} / c_{o}^{2}\right.$ in the IR limit. In this way we obtain:

$$
\psi_{l o} \lim _{k \rightarrow 0} \mathcal{P}_{l t}(k)=-\lim _{k \rightarrow 0} \mathcal{P}_{l ; 0}(k)
$$


(which is related to $w_{l t}$ ) where

$$
\lim _{k \rightarrow 0} \mathcal{P}_{l ; 0}(k)=\frac{1}{\beta \Omega} \frac{\partial \Gamma}{\partial \psi_{l o} \partial \mu}
$$

is a thermodynamic derivative by definition. We can also obtain the same results by setting $\mathbf{k}=0$ and letting $\omega \rightarrow 0$. The final result is valid for $k \rightarrow 0$, since $\mathcal{P}_{l ; 0}(k)$ is a function of $\mathbf{k}^{2}+\omega^{2} / c_{o}^{2}$ only for $k \rightarrow 0$.

We consider next the WI (3.12). Expressing again the vertex functions $\Gamma$ in terms of the $\mathcal{P}$ functions, $\Gamma_{t ; 0}=\omega \mathcal{P}_{t ; 0}$ and $\Gamma_{t ; i}=i k_{i} \mathcal{P}_{t, v}$ as in Eqs. (3.20)-(3.22), we write:

$$
\psi_{l o} \Gamma_{t t}(k)+\omega^{2} \mathcal{P}_{t ; 0}(k)+\mathbf{k}^{2} \mathcal{P}_{t ; v}(k)=0
$$

from which we obtain for $\omega=0$

$$
\lim _{\mathbf{k} \rightarrow 0} \frac{\Gamma_{t t}(0, \mathbf{k})}{\mathbf{k}^{2}}=-\frac{1}{\psi_{l o}} \lim _{\mathbf{k} \rightarrow 0} \mathcal{P}_{t ; v}\left(0, \mathbf{k}^{2}\right)
$$

(related to $z_{t t}$ ), and for $\mathbf{k}=\mathbf{0}$

$$
\lim _{\omega \rightarrow 0} \frac{\Gamma_{t t}(\omega, \mathbf{0})}{\omega^{2}}=-\frac{1}{\psi_{l o}} \lim _{\omega \rightarrow 0} \mathcal{P}_{t ; 0}(\omega, \mathbf{0})
$$

(related to $u_{t t}$ ). Note that the function $\mathcal{P}_{t ; v}$ has dimension $1-\epsilon / 2$. This suggests that the primitively divergent contribution to this function vanishes at $d_{c}$. By inspection of the PT one can verify that this is actually the case since the current insertions reduce the strength of the divergence. This fact is important since it implies the absence of singularities on the left-hand side of Eq. (3.29) (and thus the fact that $z_{t t}$ will not scale). This does not imply that $\mathcal{P}_{t ; v}$ vanishes in the limit of $k \rightarrow 0$, since the non-singular contribution can be finite.

The function $\mathcal{P}_{t ; \nu}(k)$ in the limit $k \rightarrow 0$ cannot be related directly to thermodynamic derivatives, since these involve derivatives with respect to the transverse field $\psi_{\text {to }}$. In order to identify the two limits (3.29) and (3.30), we use the following additional WI (obtained by differentiating Eq. (3.5) with respect to $A_{\mu}$ and then taking the Fourier transform):

$$
\psi_{l o} \Gamma_{t ; \mu}(k)+i k_{\nu} \Gamma_{; \nu \mu}(k)=0
$$

which in terms of the $\mathcal{P}$ functions reads (with a factor $\omega$ divided out)

$$
\psi_{l o} \mathcal{P}_{t ; 0}(k)-\Gamma_{; 00}(k)-\mathbf{k}^{2} \mathcal{P}_{; v 0}(k)=0
$$

and

$$
\begin{aligned}
& i k_{n} \psi_{l o} \mathcal{P}_{t ; v}(k)-i \omega^{2} k_{n} \mathcal{P}_{; 0 v}(k) \\
& +i k_{m}\left[\frac{k_{m} k_{n}}{\mathbf{k}^{2}} \phi_{l}(k)+\left(\delta_{m, n}-\frac{k_{m} k_{n}}{\mathbf{k}^{2}}\right) \phi_{t}(k)\right]
\end{aligned}
$$

$(m, n=1, \ldots, d)$, where a sum of repeated indices is implied and the quantity within brackets is $\Gamma_{; m n}$. Equation (3.32) for $\mathbf{k}=0$ gives

$$
\psi_{l o} \lim _{\omega \rightarrow 0} \mathcal{P}_{t ; 0}(k)=\lim _{\omega \rightarrow 0} \Gamma_{; 00}(k)=\frac{1}{\beta \Omega} \frac{\partial^{2} \Gamma}{\partial \mu^{2}},
$$

which is again a thermodynamic derivative and relates $u_{t t}$ to $\Gamma_{; 00}$. Multiplying Eq. (3.33) by $-i k_{n}$ and summing over $n$ we obtain further

$$
\psi_{l o} \mathcal{P}_{t ; v}(k)-\omega^{2} \mathcal{P}_{0 ; 0 v}(k)+\phi_{l}(k)=0,
$$

which for $\omega=0$ and $\mathbf{k} \rightarrow 0$ yields

$$
\psi_{l o} \lim _{\mathbf{k} \rightarrow 0} \mathcal{P}_{t ; v}(k)=-\lim _{\mathbf{k} \rightarrow 0} \phi_{l}(k)=-\frac{n}{m} .
$$

Here, $m(=1 / 2)$ is the mass and the last identity follows from the Galilean invariance of the action. ${ }^{4}$ Equation (3.36) relates $z_{t t}$ to $n / m$. Finally, in the limit $k \rightarrow 0$ the limit of $\Gamma_{l l}(k)$ is $(\beta \Omega)^{-1} \partial^{2} \Gamma / \partial \psi_{l o}^{2}$ by definition.

We have thus verified that, even in presence of IR divergences, the asymptotic limit of the quadratic vertex functions and of its derivatives are related to thermodynamic derivatives. Summarizing, in the IR limit one expects that $\Gamma_{l t}(k)=-\Gamma_{l ; 0}(0) \omega / \psi_{l o}$ and $\Gamma_{t t}(k)=$ $\left(2 n \mathbf{k}^{2}+\Gamma_{; 00}(0) \omega^{2}\right) / \psi_{l o}^{2}$.

Using the scaling of the RG obtained in the next Section, we obtain further that the quantities

$$
\begin{aligned}
\Gamma_{l l}(\rho k) & \sim v_{l l}(\rho), \quad \Gamma_{l t}(\rho k) \sim \omega \rho w_{l t}(\rho), \\
\Gamma_{t t}(\rho k) & \sim \rho^{2}\left[\omega^{2} u_{t t}(\rho)+\mathbf{k}^{2} z_{t t}(\rho)\right]
\end{aligned}
$$

when the RG scale $\rho$ (defined in the next section) goes to zero.

These connections between the $k \rightarrow 0$ limits of the vertex functions and the asymptotic limits of the RG couplings allow us to identify the asymptotic $\rho \rightarrow 0$ limit of the ratio

$$
\frac{c^{2}(\rho)}{c_{o}^{2}}=\frac{v_{l l}(\rho) z_{t t}(\rho)}{v_{l l}(\rho) u_{t t}(\rho)+w_{l t}(\rho)^{2}}
$$

with the macroscopic sound velocity $c_{s}$. To this end, we write

$$
\begin{aligned}
\lim _{\rho \rightarrow 0} c^{2}(\rho) & =\frac{\frac{\partial^{2} \Gamma}{\partial \psi_{l o}^{2}} \frac{1}{\psi_{l o}^{2}} \frac{n}{m}}{\frac{\partial^{2} \Gamma}{\partial \psi_{l o}^{2}}\left(-\frac{1}{\psi_{l o}^{2}} \frac{\partial^{2} \Gamma}{\partial \mu^{2}}\right)+\frac{1}{\psi_{l o}^{2}}\left(\frac{\partial^{2} \Gamma}{\partial \psi_{l o} \partial \mu}\right)^{2}} \\
& =\frac{n / m}{(d n / d \mu)_{\lambda}},
\end{aligned}
$$

which equals the square of the macroscopic sound velocity by standard thermodynamic arguments. This last identification is obtained by expressing derivatives at constant $\psi_{l o}$ in terms of physical derivatives at constant external sources $\lambda$. The ratio (3.38) enters the determinant (2.37), where it identifies the (square of the) microscopic sound velocity. The constant term $\left(v_{l l} v_{t t}-v_{l t}^{2}\right)$ vanishes identically as explained above.

Finally, we note in this context that the ratio 


$$
c_{o}^{2} \lim _{\rho \rightarrow 0} \frac{v_{l l}(\rho)}{w_{l t}(\rho)}=\frac{\frac{\partial^{2} \Gamma}{\partial \psi_{l o}^{2}}}{\frac{1}{\psi_{l o}} \frac{\partial^{2} \Gamma}{\partial \psi_{l o} \partial \mu}}=-\left.2 \psi_{l o}^{2} \frac{d \mu}{d \psi_{l o}^{2}}\right|_{\lambda}
$$

is proportional to (the inverse of) the "condensate" compressibility taken at constant external sources, which makes it a truly physical quantity. We thus expect on physical grounds that the ordinary compressibility $d n / d \mu$ and the "condensate" compressibility $d n_{o} / d \mu$ (as well as the density $n$ ) are all finite, since the physical system we are considering is in a stable phase. As a consequence, singular PT contributions will have to cancel out exactly in these particular combinations of running couplings, so that the ratios (3.38) and (3.40) as well as $z_{t t}(\rho)$ (which would give an anomalous dimension to the transverse field) will not change under the RG flow. ${ }^{13}$ In Section IV we will present a diagrammatic proof of this fact for the two couplings $u_{t t}$ and $w_{l t}$, while the argument given after Eq. (3.30) is valid to all orders in PT.

\section{FIELD-THEORETIC RG TREATMENT}

In this Section, we set up and solve the RG equations for the flow of the coupling constants discussed in the previous Sections. We will explicitly calculate the oneloop PT contributions to the relevant vertex functions, and show that higher orders of PT do not modify the IR behavior found at the one-loop level. In other words, we will be able to assess the exact IR behavior of the interacting Bose gas at zero temperature. This result contrasts with the standard (e.g., $\left.\phi^{4}\right)$ theory of critical phenomena, whereby critical exponents can be calculated only approximately. The physical origin of this simple behavior in our case stems on the fact that the same theory is free from IR divergences when formulated in the variables phase and amplitude. ${ }^{22}$ In our variables the theory remains interacting at the fixed point to give a diverging longitudinal susceptibility. Nevertheless, we will show that the effective interaction entering the transverse vertex part $\Gamma_{t t}$ vanishes exactly. This explains the difference with the $\phi^{4}$ theory in the final result for the $\mathcal{G}_{t t}$ correlation functions and recovers in an effective way the asymptotic freedom for the phase-amplitude action.

As usual, we shall discard all irrelevant running couplings of (2.34) and write equations for the four marginal couplings $\left(v_{l l}, w_{l t}, u_{t t}, z_{t t}\right)$. Before considering our specific problem, we summarize the RG procedure employed shortly. ${ }^{23,14}$

\section{A. RG procedure}

Given the set of bare running couplings $\left\{g_{i}^{o}\right\}$ of the theory (regularized in the UV with a cutoff $\Lambda$ ), we impose the following normalization conditions:

$$
\left.\Gamma_{i}(k)\right|_{k^{2}=\kappa^{2}}=g_{i} .
$$

The renormalized vertices are defined in the following way:

$$
\Gamma_{R}\left(k_{j}, g_{i}\left(g_{i}^{o}, \kappa, \Lambda\right), \kappa\right)=\Gamma\left(k_{j}, g_{i}^{o}, \Lambda\right) .
$$

Differentiating Eq. (4.2) with respect to $\kappa$ at constant $g_{i}^{o}$ we have:

$$
\left[\left.\kappa \frac{\partial}{\partial \kappa}\right|_{g}+\left.\kappa \frac{\partial g_{i}}{\partial \kappa}\right|_{g^{\circ}} \frac{\partial}{\partial g_{i}}\right] \Gamma_{R}\left(k_{i}, g, \kappa\right)=0 .
$$

If the dimension of the coupling $g_{i}$ is $\alpha_{i}$, we can introduce the dimensionless couplings $u_{i}$ and $u_{i}^{o}: g_{i}=\kappa^{\alpha_{i}} u_{i}$ and $g_{i}^{o}=\kappa^{\alpha_{i}} u_{i}^{o}$. The RG equation takes then its standard form

$$
\left[\left.\kappa \frac{\partial}{\partial \kappa}\right|_{u}+\left.\beta_{i}(u) \frac{\partial}{\partial u_{i}}\right|_{\kappa}\right]_{R}\left(k_{j}, u_{i}, \kappa\right)=0,
$$

where $\beta_{i}(u) \equiv \kappa\left(\partial u_{i} / \partial \kappa\right)_{g^{\circ}}$. One can then verify that the following equation holds:

$$
\Gamma_{R}\left(\rho k_{i}, u, \kappa\right)=\rho^{\alpha} \Gamma_{R}\left(k_{i}, u(\rho), \kappa\right),
$$

where $\rho d u_{i}(\rho) / d \rho=\beta_{i}(u)$ and $\alpha$ is the bare dimension of the vertex function. To obtain the IR behavior of a vertex function, it suffices to scale it with its bare dimension $\alpha$ and to substitute the new value of the running coupling $u(\rho)$. In particular, when the normalization condition is $\left.\Gamma_{R}(k, \kappa)\right|_{k=\kappa}=g_{i}=\kappa^{\alpha_{i}} u_{i}(1)$ we obtain:

$$
\Gamma_{R}\left(\rho k_{i}, u, \kappa\right)=\kappa^{\alpha_{i}} \rho^{\alpha_{i}} u_{i}(\rho) .
$$

In this way, if near the fixed point the running coupling is flowing to zero with an exponent $y_{i}\left[u_{i}(\rho) \sim \rho^{y_{i}}\right]$, the vertex part will have the following IR behavior:

$$
\Gamma_{R}(k) \sim k^{\alpha_{i}+y_{i}} .
$$

It is thus simple to convert the $\rho$-behavior of the running couplings into the $k$-behavior of the relative vertex functions. The simple relation between the renormalized couplings and the vertex functions has been used to identify the $k \rightarrow 0$ limit of $\Gamma_{i j}(k)$ given before (3.37).

\section{B. Additional constraints on the running couplings}

We now proceed to write four RG equations for the left four couplings: $v_{l l}, w_{l t}, u_{t t}$, and $z_{t t}$. All other couplings can be readily obtained from them. Indeed, the condensate $\psi_{l o}$ entering the WI is the exact condensate density which is not scaling. The other marginal couplings $v_{l t t}$ and $v_{t t t t}$ have been eliminated in favour of $v_{l l}$ through Eqs. (3.18) and (3.19).

It turns out that also the four left running couplings left are not renormalizing independently. As it was argued at the end of Section IIIB, we expect that 
the combinations of couplings $z_{t t}, w_{l t} / v_{l l}$, and $c^{2}(\rho)=$ $v_{l l} z_{t t} /\left(v_{l l} u_{t t}+w_{l t}^{2}\right)$ are invariant of the RG flow. Here, we show how these properties for $w_{l t} / v_{l l}$ and $c$ can be proved by analyzing the PT. These identities are shown to hold at one-loop level in Appendix C. The coupling $z_{t t}$ has to remain constant for the argument given after Eq. (3.30). The absence of divergences in this case follows directly from the WI and power counting. We now proceed to prove the other two identities by analysis of the PT to all orders.

The coupling $w_{l t}$ can be identified with $v_{l l}$ via the WI (3.13) and (3.14), which relate $w_{l t}$ to $\Gamma_{t t ; 0}$ and $v_{l l}$ to $\Gamma_{l t t}$, respectively. By inspection of the leading singular terms to all orders in perturbation theory, $\Gamma_{t t ; 0}$ and $\Gamma_{l t t}$ are then found to be proportional to each other. As a matter of fact, the only way in which we can make an insertion of the longitudinal $(l)$ leg is to use the bare interaction term $v_{l t t}$, since it connects the external longitudinal leg to two internal transverse legs. It is then clear that this corresponds to performing a density insertion with the most relevant term (since $|\psi|^{2}=\psi_{t}^{2}+\psi_{l}^{2}+2 \psi_{l o} \psi_{t}+\psi_{l_{o}}^{2}$ ), apart from the value of the bare external insertion that is different from the bare $v_{l t t}$. (See Appendix A for a discussion on the density fields and the associated couplings.) Diagrammatically this reads:

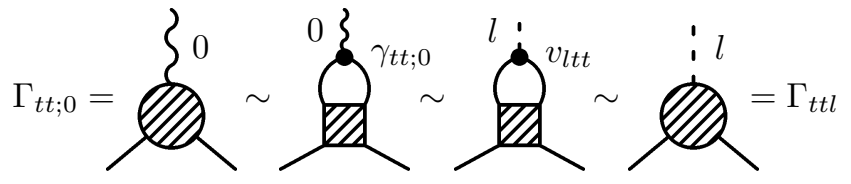

We can write the chain of identities in the following way (by $\sim$ we mean the leading-order behavior as $k \rightarrow 0$ ):

$$
\begin{aligned}
& \psi_{l o} \Gamma_{l l}\left(k_{0}\right) \stackrel{(\mathrm{WI})}{\sim} \Gamma_{l t t}\left(0, k_{0}\right) \stackrel{(\mathrm{PT})}{\sim} \frac{v_{l t t}}{\gamma_{t t ; 0}} \Gamma_{t t ; 0}\left(0, k_{0}\right) \\
& \stackrel{\mathrm{WI}}{\sim} \frac{v_{l t t}}{\gamma_{t t ; 0}} \frac{\Gamma_{l t}\left(k_{0}\right)}{k_{0}}
\end{aligned}
$$

where by $\gamma_{t t ; 0}$ we indicate the perturbative insertion corresponding to $\Gamma_{t t ; 0}$ that in our case is $w_{l t}$ (see Appendix A). In Eq. (4.9) we have set the spatial part to zero exploiting the hypothesis of singular dependence through only $k_{0}^{2}+\mathbf{k}^{2}$. We thus conclude that, at the leading order in the divergent contributions:

$$
\Gamma_{l l}\left(k_{0}\right) \sim \frac{v_{l l}}{w_{l t}} \frac{\Gamma_{l t}\left(k_{0}\right)}{k_{0}} .
$$

In this way, the logarithmic divergences entering in the RG equation for $v_{l l}$ must also appear in the RG equation for $w_{l t}$. If the exact (to all orders) RG equation for $v_{l l}$ has the following form

$$
\rho \frac{d v_{l l}}{d \rho}=f\left(v_{l l}, w_{l t}, u_{t t}\right),
$$

the resulting equation for $w_{l t}$ will be after a transient region:

$$
\rho \frac{d w_{l t}}{d \rho}=f\left(v_{l l}, w_{l t}, u_{t t}\right) \frac{w_{l t}}{v_{l l}} .
$$

Dividing Eq. (4.11) by Eq. (4.12) we thus get:

$$
\frac{d w_{l t}}{d v_{l l}}=\frac{w_{l t}}{v_{l l}}
$$

impling

$$
\frac{v_{l l}(\rho)}{w_{l t}(\rho)}=\text { constant } \equiv C_{1} .
$$

In a similar way, the invariance of $c(\rho)$ follows from the exact connection between the singular parts of $\Gamma_{; 00}$ and $\Gamma_{l l}$, associated respectively with $u_{t t}$ and $v_{l l}$ :

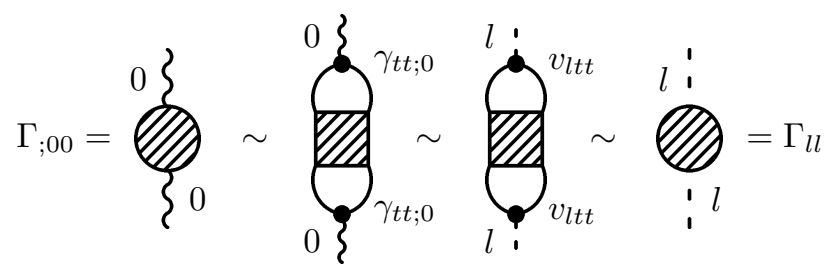

This procedure leads to the following asymptotic equation for $u_{t t}$

$$
\rho \frac{d u_{t t}}{d \rho}=-f\left(v_{l l}, w_{l t}, u_{t t}\right) \frac{w_{l t}^{2}}{v_{l l}^{2}} .
$$

Using Eq. (4.14) we obtain $d u_{t t} / d v_{l l}=-C_{1}^{-2}$ and

$$
u_{t t}(\rho)=-C_{1}^{-2} v_{l l}(\rho)+C_{2},
$$

so that $u_{t t}+w_{l t}^{2} / v_{l l}=C_{2}$ and $c(\rho)$ is invariant (cf. Eq. (3.38)). The values of the two constants $C_{1}$ and $C_{2}$ can be extracted from the $\rho \rightarrow 0$ limit of the running couplings, specifically from Eqs. (3.39) and (3.40). The flow of the four running couplings will satisfy thus these three identities. We are left eventually with only one independent running coupling, for instance, $v_{l l}$.

The constraints found above for $v_{l l}, w_{l t}, z_{t t}$, and $u_{t t}$ fix completely the IR behavior of two out of three Green's functions, say $\mathcal{G}_{l t}(k)$ and $\mathcal{G}_{t t}(k)$. From Eq. (2.36) one can in fact say that the expressions for $\mathcal{G}_{l t}(k)$ and $\mathcal{G}_{t t}(k)$ at vanishing $v_{l t}$ and $v_{t t}$ involve only the above combinations of running couplings, which do not scale and are related to physical quantities. The resulting exact IR behavior is thus given by:

$$
\begin{aligned}
& \mathcal{G}_{t t}(\mathbf{k}, \omega)=-\frac{c^{2} m n_{o}}{n} \frac{1}{\omega^{2}+c^{2} \mathbf{k}^{2}} \\
& \mathcal{G}_{l t}(\mathbf{k}, \omega)=-\frac{m c^{2}}{2 n} \frac{d n_{o}}{d \mu} \frac{\omega}{\omega^{2}+c^{2} \mathbf{k}^{2}} .
\end{aligned}
$$


This result is valid at all orders of perturbation theory and does not depend on the scaling of $v_{l l}$. The Goldstone mode is accordingly stabilized only by symmetry requirements, irrespective of the behavior of $v_{l l}$. The vanishing of $v_{l l}\left(\sim \Sigma_{12}\right)$ is no longer changing the IR behavior of $\mathcal{G}_{t t}$ and $\mathcal{G}_{l t}$. As discussed in the Introduction, the IR behavior of $\mathcal{G}_{l l}$ is expected to depend on dimensionality, and thus cannot be fixed by symmetry requirements only.

\section{One-loop equation for $v_{l l}$}

To proceed further we need to perform a specific calculation of the RG equation for one coupling, for instance $v_{l l}$. At the one-loop level, only one diagram contributes to $\Gamma_{l l}$ (see Appendix B). (Derivation of the other oneloop equations is not required. For comparison, we give the full set of one-loop equations in Appendix $\mathrm{C}$ for the marginal running couplings entering the propagators.) With dimensional regularization and after the $\epsilon$ expansion has been carried out, $\Gamma_{l l}$ reads:

$$
\Gamma_{l l}(k)=v_{l l}-\frac{v_{l l}^{2} \kappa^{-\epsilon}}{2 \psi_{l o}^{2} z_{t t}^{2} \epsilon}
$$

where $\kappa^{2}=\mathbf{k}^{2}+k_{o}^{2}$ and the factor $K_{d}=2 /(4 \pi)^{d / 2} \Gamma(d / 2)$ has been absorbed in the definition of the running coupling. We made use of Eq. (3.18) to connect $v_{l t t}$ to $v_{l l}$. To set up the RG equations, we need to define dimensionless running couplings (see Section IV A). Since all quadratic running couplings are already dimensionless (at any dimension), only the condensate density $\psi_{l o}$ needs to be scaled by a factor $\kappa^{\epsilon / 2}$ :

$$
\bar{\psi}_{l o}=\psi_{l o} \kappa^{\epsilon / 2} .
$$

Note that the powers of $c_{o}$ have already been eliminated in $\psi_{l o}$ which is redefined like $c_{o}^{-3 / 2} \psi_{l o}$. By minimal subtraction of the $1 / \epsilon$ pole one obtains the following $\mathrm{RG}$ equation for $v_{l l}(\rho)$ :

$$
\rho \frac{d v_{l l}}{d \rho}=\frac{v_{l l}^{2}}{2 \psi_{l o}^{2}(\rho) z_{t t}^{2}},
$$

where $\psi_{l o}(\rho)=\psi_{l o}(1) \rho^{\epsilon / 2}$ exactly while $z_{t t}$ is invariant. We stress that the scaling of $\psi_{l o}$ is a trivial effect of its dimension and the condensate density is not renormalizing. An alternative procedure would be to fix the condensate density and use scale dependent WI in order to connect the running couplings $v_{l l}, v_{l t t}$, and $v_{t t t t}$. The solution of Eq. (4.22) is straightforward and depends on dimensionality. For $d=3, \psi_{l o}$ is constant and

$$
\frac{v_{l l}(\rho)}{v_{l l}(1)}=\left[1-\frac{v_{l l}(1)}{2 \psi_{l o}(1)^{2} z_{t t}(1)^{2}} \ln \rho\right]^{-1} .
$$

For $d<3$ we obtain instead:

$$
\frac{v_{l l}(\rho)}{v_{l l}(1)}=\left[1+\frac{v_{l l}(1)}{2 \psi_{l o}(1)^{2} z_{t t}(1)^{2}} \frac{\rho^{-\epsilon}-1}{\epsilon}\right]^{-1} .
$$

We recall that the $R G$ equations are valid only after a transient region, so that the boundary conditions for Eq. (4.22) should involve the running couplings at some intermediate value $0<\bar{\rho}<1$. For simplicity, we write the solution of this equation in terms of the coupling at $\rho=1$. Since the results do not depend on the boundary conditions (provided the sign is not changed), we assume that the $\rho=1$ is the point where the RG equations start being accurate.

In both cases (4.23) and (4.24) the coupling $v_{l l}$ is flowing to zero, implying the vanishing of $\Sigma_{12}(k \rightarrow 0)$ (here $\left.\Sigma_{12}=\left[\Gamma_{l l}-\Gamma_{t t}+i\left(\Gamma_{l t}+\Gamma_{t l}\right)\right] / 4\right)$. At the same time, the constraint on the other couplings implies that $w_{l t}(\rho)$ vanishes like $v_{l l}(\rho)$, which from Eq. (3.38) gives that $u_{t t}(\rho)$ flows to $z_{t t}(\rho) c^{2}(\rho) / c_{o}^{2}$. Since both $c(\rho)$ and $z_{t t}(\rho)$ are constants of motion of the RG flow, $u_{t t}(\rho) \rightarrow z_{t t}(1)$. We can thus find the asymptotic expression for the longitudinal Green's functions as follows:

$$
\mathcal{G}_{l l}(k)=-\frac{1}{v_{l l} u_{t t}} \frac{u_{t t} k_{0}^{2}+z_{t t} \mathbf{k}^{2}}{k_{0}^{2}+\left(c^{2} / c_{o}^{2}\right) \mathbf{k}^{2}} \rightarrow-\frac{1}{v_{l l}} .
$$

According to the scaling (4.7) of the RG, this implies that

$$
\mathcal{G}_{l l}(\rho k) \sim \frac{1}{v_{l l}(\rho)} .
$$

The above result, together with Eqs. (4.18) and (4.19), gives the complete IR behavior for all one-particle Green's functions. By using the results of Section III on the finite value of the small $k$-limit of the vertex functions, it is possible to rewrite the result (4.26) in terms of thermodynamical quantities:

$$
\begin{aligned}
\mathcal{G}_{l l}(\mathbf{k}, \omega)= & \frac{c^{2}}{8 n_{0} n}\left(\frac{d n_{0}}{d \mu}\right)^{2} \frac{\omega^{2}}{\omega^{2}+c^{2} \mathbf{k}^{2}} \\
+ & \begin{cases}\frac{c_{o} n_{o}}{64 \pi^{2} n^{2}} \ln (k / \Lambda) & (d=3) \\
-\frac{c_{o} n_{o} K_{4-\epsilon}}{8 n^{2} \epsilon k^{\epsilon}} & (1<d<3)\end{cases}
\end{aligned}
$$

where $\Lambda$ is an ultraviolet cutoff of the order of the sound velocity (corresponding to the region where the RG starts to be accurate) and $c_{o}$ is the sound velocity at the scale $\Lambda$. After that point, $c(\rho)$ is, to a good accouracy, an invariant of the RG flow, so that we can substitute the fixed point value $c_{o}=c$.

We note that for $d=1(\epsilon=2)$ the transverse and longitudinal divergences become identical while the condensate vanishes. It is thus expected that transverse and longitudinal fluctuations become symmetric since it is no longer possible even to define longitudinal and transverse directions. The main assumptions of our approach will thus break down, indicating the existence of a lower critical dimension. Nevertheless, since the results are valid exactly for $1<d<3$, as said already, the predicted IR behavior approaches the correct result for $d \rightarrow 1 .^{9}$ 
The usual Green's functions can be readily obtained from Eqs. (4.18), (4.19) and (4.27):

$$
\begin{aligned}
\mathcal{G}_{11}(\mathbf{k}, \omega)=- & \frac{c^{2} m n_{o}}{n} \frac{1}{\omega^{2}+c^{2} \mathbf{k}^{2}}+\frac{m c^{2}}{n} \frac{d n_{o}}{d \mu} \frac{i \omega}{\omega^{2}+c^{2} \mathbf{k}^{2}} \\
& +\mathcal{G}_{l l}(\mathbf{k}, \omega), \\
\mathcal{G}_{12}(\mathbf{k}, \omega)= & \frac{c^{2} m n_{o}}{n} \frac{1}{\omega^{2}+c^{2} \mathbf{k}^{2}}+\mathcal{G}_{l l}(\mathbf{k}, \omega),
\end{aligned}
$$

where we have restored the canonical dimensions and reintroduced the mass $m$. The leading order of the expressions (4.28) (i.e., the first term) coincides with that found by Gavoret and Nozières. ${ }^{4}$ The additional terms proportional to $\mathcal{G}_{l l}$ give the next-to-leading contribution to the Green functions coming from the divergent diagrams. These diverging sub-leading contributions were identified for the first time in Ref. 8 without calculating the coefficients. Later, by improving the Bogoliubov approximation and exploiting the analogy with spin-wave theory, Weichman found an explicit expression for $\mathcal{G}_{l l}$ valid for the weak-coupling Bose gas. ${ }^{15}$ Assuming a Landau quantum-hydrodynamic Hamiltonian, the authors of Ref. 24 have derived the same expression given by (4.27) for the longitudinal correlation function.

\section{Extension to all orders of PT}

Although the IR behavior of $\mathcal{G}_{i j}$ has been found only at the one-loop order, we will show now that it is actually correct to all orders. We begin by studying the nature of the fixed point. The scaling of the running couplings near the fixed point is given by Eqs. (4.23) and (4.22) for $v_{l l}$ and by the WI for $v_{l t t}$ and $v_{t t t t}$ :

$$
\begin{aligned}
v_{l t t}(\rho) & =v_{l l}(\rho) / \psi_{l o}(\rho) \sim v_{l l}(\rho) \rho^{-\epsilon / 2} \\
v_{t t t t}(\rho) & =3 v_{l t t}(\rho) / \psi_{l o}(\rho) \sim v_{l l}(\rho) \rho^{-\epsilon} .
\end{aligned}
$$

One thus finds that $v_{l t t}$ always vanishes near the fixed point. For $d=3 v_{t t t t}$ flows also to zero, while for $d<3$ it flows to a finite fixed point value $v_{t t t t}^{*}$. We can thus draw one important conclusion, namely that for $\epsilon=0$ the theory is asymptotically free. Marginal interactions become marginally irrelevant (they vanish like $1 / \ln \rho$ ) and the results obtained at one loop are exact, in agreement with Benfatto calculations. ${ }^{16}$

Unfortunately, for $\epsilon>0$ the theory is not free since $v_{t t t t}$ flows towards a finite value. This fact may, in principle, invalidate the results found at one-loop level, because higher-order terms are not negligible near the fixed point. Note, however, that contrary to what happens in critical phenomena, the asymptotic behavior of $v_{l l}$ does not depend on the position of the fixed point $\left(v_{t t t t}^{*}\right)$ but only on its very existence. This can be verified from Eq. (4.30). When $v_{t t t t}(\rho \rightarrow 0)$ is finite, $v_{l l}(\rho) \sim \rho^{\epsilon}$ and the exponent does not depend on the values of interactions. For this reason it is possible to establish the scaling of $v_{l l}$ without a precise knowledge of the corresponding $\mathrm{RG}$ equation.
Actually its "form" is enough, as we shall argue and explain in the following by analyzing the PT. One has to prove either that $v_{l l}(\rho) \sim \rho^{\epsilon}$, or the existence of the fixed point, since the two statements are equivalent. We will prove the former statement in the following.

Let us consider the following two-loops diagrams contributing to $\Gamma_{l l}$ :

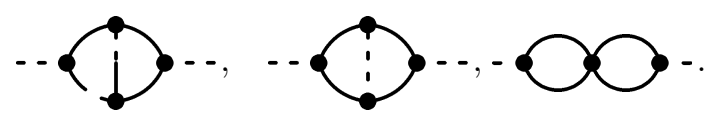

These diagrams, once expanded in $\epsilon$, develop $1 / \epsilon$ poles, and thus they contribute to the equation for $v_{l l}(\rho)$. Without explicit calculations, we can infer the form of this contributions. We have seen that for $\rho \rightarrow 0, \mathcal{G}_{t t}(k)$ and $\mathcal{G}_{l t}(k)$ do not depend on the explicit scaling of $v_{l l}$, thus they will not contribute to (4.31) with factors $v_{l l}$ (at least at leading order as $\rho \rightarrow 0$ ). This means, in particular, that the first diagram contributes to the beta-function only with a term proportional to $v_{l t t}^{4}$, coming from the vertices. Its contribution is thus negligible with respect to the one-loop contribution (4.22). These classes of diagrams do not affect the RG equations.

Let us consider now the second diagram in (4.31), for which the situation is different since $\mathcal{G}_{l l}$ is proportional to $1 / v_{l l}(\rho)$, but each internal $\mathcal{G}_{l l}$ must appear in combination with two $v_{l t t}$. From the scaling form at one-loop level, that we assume valid for the moment, we find that as $\rho \rightarrow 0$ :

$$
v_{l t t}^{2}(\rho) \mathcal{G}_{l l}(k) \sim-\frac{v_{l t t}^{2}(\rho)}{v_{l l}(\rho)} \sim-\frac{v_{t t t t}^{*}}{3} .
$$

Taking into account Eq. (4.32) this implies that the form the contribution of the second diagram in (4.31) is of the same form of (4.22), i.e., it has exactly the same $\rho$ dependence. It does thus not change the form of the solution for $v_{l l}$, even though it can give a multiplicative factor in the final scaling.

The last diagram in (4.31) has a behavior similar to the second one. It depends on $v_{l l}$ only through the two $v_{l t t}$ connected to the external $\mathcal{G}_{l l}$. The internal part gives a finite contribution in $\rho$ and again, once added to (4.22), it does not change the scaling of $v_{l l}$. We thus conclude that the contributions of all two-loops diagrams to (4.22) do not change the IR behavior of $v_{l l}$.

The same arguments can be extended at higher order in loops. The structure of the PT remains the same. The presence of even only one $\mathcal{G}_{l t}$ is enough to suppress the diagram due to the $v_{l t t}$ non compensated by any $1 / v_{l l}$. The $\mathcal{G}_{l l}$ appears always in combination with two $v_{l t t}$, giving a finite non-dangerous term. All diagrams thus are either negligible, or give a finite contribution for $\rho \rightarrow 0$ multiplied by two $v_{l t t}$ coming from the two vertices connected to the external lines.

The RG equation for $v_{l l}$ to all orders has thus the following simple form: 
$\rho \frac{d v_{l l}}{d \rho}=\frac{v_{l l}^{2}}{\psi_{l o}^{2}(\rho)}\left[A_{0}+A_{1} v_{t t t t}^{*}+A_{2}\left(v_{t t t t}^{*}\right)^{2}+\ldots\right]$

Provided the sum of the series does not change sign with respect to the sign of the first term, for $\rho \rightarrow 0$ we obtain:

$$
v_{l l}(\rho)=\frac{\psi_{l o}^{2}(a)}{A\left(v_{t t t t}^{*}\right)} \epsilon \rho^{\epsilon}
$$

where $a<1$ is the value of $\rho$ for which the leading behavior established by (4.33) holds and $A\left(v_{t t t t}^{*}\right)$ is the sum of the series of Eq. (4.33). This concludes our proof. Higher-order diagrams do not change the IR behavior, as they only can affect the finite coefficients.

We have thus proved that the scaling (4.24) holds apparently for any value of $\epsilon>0$. One has to recall that the procedure breaks down at $d=1$, since the condensate vanishes in that case, corresponding to $\epsilon<2$. In conclusion, we have proved that the IR behavior found at one loop is maintained for $1<d \leq 3$.

\section{E. A non-trivial fixed point without anomalous dimensions}

We have shown that the fixed point is characterized by a finite value of the interacting constant among transverse fluctuations, similarly to what happens for the $\phi^{4}$ theory. We have also shown that no anomalous dimension appears in the transverse correlation function. This is clearly due to the constraints originating from the WI. The question is now how these constraints can be implemented in the structure of the perturbation theory. Actually, the same diagrams responsible for the anomalous dimension in the $\phi^{4}$ theory are also present here in $\Gamma_{t t}$. For instance:

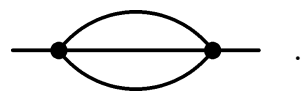

These diagrams, however, do not contribute in practice because other diagrams contribute with opposite sign and remove the divergence. This can be realized to all orders by looking at the form of Eq. (4.32). The structure $\mathcal{G}_{l l} v_{l t t}^{2}$ acts as an effective $v_{t t t t}$ interaction mediated by the longitudinal field. According to Eq. (4.32), it has the opposite sign with respect to the fixed point interaction $v_{t t t t}^{*}$. All contributions to the effective interaction are given by the following graphical equation:

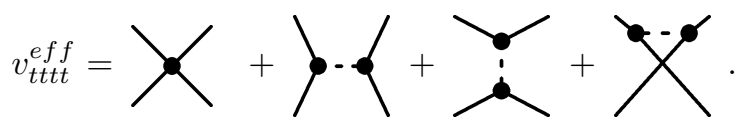

Evaluating the diagrams entering Eq. (4.36), one finds that at the fixed point $v_{t t t t}^{e f f}$ vanishes due to the exact cancellation of the fixed point $v_{t t t t}^{*}$ with the interaction mediated by $\mathcal{G}_{l l}$.
This explains why no $\phi^{4}$-like divergences appear in the contribution to $\Gamma_{t t}$. This does not mean, however, that the theory is free at the fixed point. As a matter of fact, $v_{t t t t}^{e f f}$ is not defined in terms of one-particle irreducible diagrams for $\Gamma_{t t t t}$. Thus the above cancellation is only partial in some diagrams for $\Gamma_{l l}$. This can be seen by expanding in a typical diagram for $\Gamma_{l l}$ the definition of $v_{t t t t}^{\text {eff }}$ (represented here by the dashed blob):

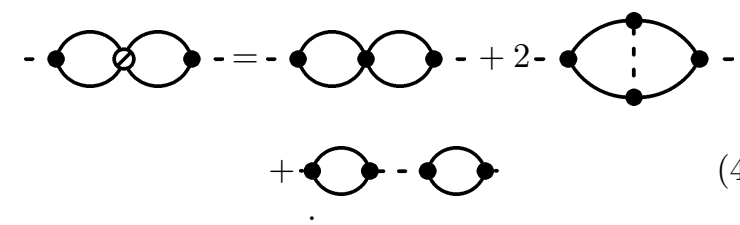

The above diagram vanishes, but the last diagram does not contribute to $\Gamma_{l l}$ since it is not one-particle irreducible. Thus, $v_{t t t t}$ does affect the equation for $v_{l l}$ even if it gives only finite renormalization, as shown before. On the other side, one can verify that all $v_{t t t t}$ terms appearing at all orders in $\Gamma_{t t}$ are exactly canceled by the mediated interaction. We have thus explicitly shown how the diagrams responsible for the appearance of the anomalous dimension in $\phi^{4}$ theory get eliminated in the present case. This is the counterpart of what happens in the phase-amplitude variables, where the integration of the amplitude fields reconstructs an effective full-gaugeinvariant action.

\section{F. IR behavior of the the response functions}

In Section IV C we have found the IR behavior of the quadratic Green's functions. Only $\mathcal{G}_{l l}$ is affected by the nontrivial scaling of the four quadratic running couplings. We found that the form of the spectrum is unaffected by the RG flow. In the same way, we find that the physical response functions, specifically the density-density, density-current, and current-current response functions $\left(\mathcal{G}_{\mu, \nu}(k)\right)$ depend only on RG-invariant combinations of the running couplings. This is not surprising, since the expressions found for these functions in the limit $k \rightarrow 0$ by Gavoret and Nozières depend only on two finite physical quantities, $n$ and $c^{2}$. As an example, we consider explicitly the density-density correlation function $\mathcal{G}_{; 00}$. We obtain its expression from the general relation:

$$
\mathcal{G}_{; \nu \mu}(p)=\Gamma_{; \nu \mu}(p)+\Gamma_{j ; \nu}(-p) \mathcal{G}_{i j}(-p) \Gamma_{i ; \mu}(p)
$$

with $\mu=\nu=0$. The scaling of the composite $\Gamma$ functions has been linked to the quadratic running couplings through the WI (3.26) and (3.28):

$$
\Gamma_{l ; 0}(\mathbf{k}, \omega) \sim w_{l t} n_{0}^{1 / 2} \quad, \quad \Gamma_{t ; 0}(\mathbf{k}, \omega) \sim u_{t t} n_{0}^{1 / 2} \omega .
$$

Substituting this expression into Eq. (4.38) and using $u_{t t} \rightarrow-\Gamma_{; 00} / n_{o}$ in the IR, we recover the GavoretNozières result (with the mass reinserted explicitly): 


$$
\mathcal{G}_{; 00}(p)=-\frac{n \mathbf{k}^{2} /\left(n_{0} m\right)}{\omega^{2}+c^{2} \mathbf{k}^{2}} .
$$

No anomalous terms appear. The same is true for the other response functions.

\section{CONCLUSIONS}

Infrared divergences plague perturbation theory of the interacting Bose gas without the presence of a true physical instability for the system. This means that IR divergences must disappear from the final physical quantities, like the thermodynamical derivatives. However, it is not trivial to control the divergent parts of the perturbation theory whithin approximated shemes. Divergences are due to the strong fluctuations of the phase of the brokengauge-symmetry order parameter. In this paper, we have set up a RG approach to treat these singularities in a systematic way. Since they must disappear in the final results, most divergences are actually related. We implemented these connections through Ward identities. We found that WI are sufficient to guarantee the stability of the superfluid phase with respect to phase fluctuations. As a consequence, the sound velocity of the macroscopic and microscopic excitations is an invariant of the RG flow.

Nevertheless the divergences are not totally ineffective. The WI allow to reduce the set of independent running couplings to a single one, for which we have derived a flow equation at the one-loop order. Within this approximation, we found that the theory becomes free at $d=3$, i.e., all running couplings flow to zero in agreement with Benfatto result. ${ }^{16}$ One-loop order proves thus enough to obtain the exact result at $d=3$, that gives a logarithmically divergent longitudinal correlation function $\mathcal{G}_{l l}$. For $1<d<3$ the fixed point is characterized by a finite value of the coupling $v_{t t t t}$ among transverse fluctuations, being a truly interacting fixed point. Even in this case we found that the one-loop result gives the correct exponent for $\mathcal{G}_{l l}$. Higher-order diagrams are not negligible like as in the $d=3$ case, but they contribute like the one-loop ones, and thus they do not change the IR behavior. Concerning the two other correlation functions $\mathcal{G}_{l t}$ and $\mathcal{G}_{t t}$, we found that their IR behavior is determined by WI and no anomalous dimension appears. This result can be surprising for the transverse one, since at the fixed point the interaction is finite and an anomalous dimension could appear. We showed, however, how the structure of the $\mathrm{PT}$ adapts in a nontrivial way near the fixed point, in order to keep $v_{t t t t}$ finite and fulfill WI. The transverse field is effectively free once one adds to $v_{t t t t}$ the interaction mediated by the longitudinal fields.

In conclusion, our approach has enabled us to prove, within a coherent frame, the disappearance of the divergences in the physical quantities while keeping a firm control on singularities during the procedure. The nontrivial evolution of the structure of the propagator from large momenta (Bogoliubov) to small momenta (fixed point) can be readily followed in terms of the flow of the running couplings. This approach can be useful, in general, to study stable systems where singularities appears due to a broken continuous symmetry without true instabilities.

We acknowledge discussions with $\mathrm{Ph}$. Noziéres, G. Benfatto, and M. Grilli. F.P. gratefully acknowledges Europa Metalli S.p.A. for partial financial support and the Statistical Mechanics and Complexity (SMC) center in Rome for hospitality in the last stage of this work.

\section{APPENDIX A: GAUGE INVARIANCE CONSTRAINTS FOR THE RUNNING COUPLINGS}

The introduction in Eq. (2.34) of the couplings associated with the monomials of the $\chi_{l / t}$ fields may lead to some difficulties in defining gauge invariance itself. Since the theory we are constructing must satisfy the original gauge invariance defined in Eq. (3.1) in terms of the $\psi$ fields, we need to specify the the equivalent transformation for the action (2.34) in terms of the new fields. In this way, we can establish in a different way a connection among the bare couplings of the theory.

To discuss this point we proceed by steps. We begin with the global part of the action and introduce the following simplified notation for the couplings:

$$
S=\sum_{n, m} \frac{v_{n, m}}{m ! n !} \psi_{l}^{n}(x) \psi_{t}^{m}(x)+\lambda_{l}\left(\psi_{l}+\alpha_{l}\right)+\alpha_{t}\left(\psi_{t}+\alpha_{t}\right) .
$$

In this expression, $\alpha_{l / t}$ are two arbitrary real numbers. The global gauge invariance in terms of these fields reads:

$$
\left[\begin{array}{l}
\psi_{l}^{\prime}(x) \\
\psi_{t}^{\prime}(x)
\end{array}\right]=\mathbf{R}(\chi)\left[\begin{array}{l}
\psi_{l}(x)+\alpha_{l} \\
\psi_{t}(x)+\alpha_{t}
\end{array}\right]-\left[\begin{array}{c}
\alpha_{l} \\
\alpha_{t}
\end{array}\right]
$$

where $\mathbf{R}$ is defined in Eq. (3.3) and the external fields change as follows:

$$
\left[\begin{array}{c}
\lambda_{l}^{\prime}(x) \\
\lambda_{t}^{\prime}(x)
\end{array}\right]=\mathbf{R}\left[\begin{array}{c}
\lambda_{l}(x) \\
\lambda_{t}(x)
\end{array}\right]
$$

Note that the transformation (A2) depends on $\alpha_{l / t}$. This is due to the fact that the new action is no longer invariant under rotations around the origin in the space $\left(\psi_{l}, \psi_{t}\right)$, but rather around the point $\left(-\alpha_{l},-\alpha_{t}\right)$. It is also clear the the original action is manifestly gauge invariant, since it is a sum of gauge invariant terms (essentially $|\psi|^{2 n}$ ). By contrast, the action (A1) is invariant under the transformations (A2)-(A3) only for specific values of the set of couplings $\left\{v_{n m}\right\}$. It is easy to find these constraints by implementing the transformation (A2)-(A3) infinitesimally: 


$$
\left\{\begin{array}{l}
\delta \psi_{l}^{\prime}(x)=-\left(\psi_{t}(x)+\alpha_{t}\right) \delta \chi \\
\delta \psi_{t}^{\prime}(x)=-\left(\psi_{l}(x)+\alpha_{l}\right) \delta \chi
\end{array} .\right.
$$

Imposing the invariance of $S$ given by (A1) under the transformation (A4) gives the following set of equations (with $\alpha_{t}=0$ ):

$$
-m v_{n+1, m-1}+n v_{n-1, m+1}+\alpha_{l} v_{n, m+1}=0 .
$$

One can readily verify that these conditions coincide with the WI for $k_{i}=0$, the two procedures being equivalent.

At this point it is easy to establish a link between the old couplings $\{\mu, v\}$ and the new ones $\left\{v_{n, m}\right\}$. By using Eq. (A5) repeatedly, we find a relation among all the running couplings and reduce the whole set to the independent ones. This can be shown by noticing that all the running couplings can be derived by knowing only $v_{n, 0}$ for all $n$, since the coupling $v_{n, m}$ is related to $v_{n-1, m}$ and $v_{n+1, m-2}$ by (A5). Thus, for any positive integer $k$ we can draw a line in the $n-m$ plane that starts at $m=0$ and $n=k$ and end at $m=2 k$ and $n=0$. If the running couplings, identified by the pair $(n, m)$, which lye between the axes and this line are specified, then given $v_{k+1,0}$ one can obtain all the couplings along the line $v_{n,-2 n+2 k}$ for $0 \leq n \leq k+1$, thus extending the portion of the plane where the $v_{n, m}$ are known. Proceeding in this way, all the running couplings can be obtained if $v_{k, 0}$ is known. Thus $v_{k, 0}$ can be chosen to be the independent variable. The usual action is recovered by choosing $v_{1,0}=-2 \alpha_{l}\left(\mu-v \alpha_{l}^{2}\right), v_{2,0}=-2\left(\mu-3 v \alpha_{l}^{2}\right)$, and all others $v_{n, 0}=0$ (for $\left.\alpha_{t}=0\right)$. All other couplings can be found by the use of (A5).

We consider now the local gauge invariance. The invariance (3.1) holds for the original action. We need to clarify the precise meaning of requiring that the action written in terms of the new couplings $v_{n, m}$ is invariant under (3.1). First, we face the problem of defining a $\mu$ coupling, since there is no more trace of it in the new action (A1). The implementation of the invariance (A5) will thus involve a more complicated transformation of the fields, since no simple coupling couples to $|\psi|^{2}$. To find the proper transformation of the couplings, we need to consider the transformation under an infinitesimal local gauge transformation of the derivative term:

$$
\begin{aligned}
& \delta\left[i w_{l t} \int d x \psi_{t}(x) \partial_{\tau} \psi_{l}(x)\right]= \\
& \quad-i \frac{w_{l t}}{2} \int d x\left[\psi_{l}^{2}+\psi_{t}^{2}+2 \alpha_{l} \psi_{l}\right] \partial_{\tau} \delta \chi(\tau) .
\end{aligned}
$$

This implies the following transformation (with $\alpha_{t}=0$ ):

$$
\left\{\begin{array}{l}
v_{2,0} \rightarrow v_{2,0}-i w_{l t} \partial_{\tau} \delta \chi(\tau) \\
v_{0,2} \rightarrow v_{0,2}-i w_{l t} \partial_{\tau} \delta \chi(\tau) \\
v_{1,0} \rightarrow v_{1,0}-i w_{l t} \alpha_{l} \partial_{\tau} \delta \chi(\tau) .
\end{array}\right.
$$

One can readily recognize in (A6) the field $|\psi|^{2}$ written in terms of the new variables. An equivalent approach to define the local $\tau$-gauge invariant is then to introduce a new external source $\mu_{\text {ext }} w_{l t} / 2$ that couples to $|\psi|^{2}$ (which will be set to zero at the end of the calculation). In term of $\mu_{\text {ext }}$, gauge invariance is defined as usual by $\mu_{\text {ext }} \rightarrow \mu_{\text {ext }}+\partial_{\tau} \chi(\tau)$.

We consider now the gauge transformations involving spatial variations. In that case we have the following gradient terms in the action

$$
z_{t t} \int d x\left|\nabla \psi_{t}\right|^{2}+z_{l l} \int d x\left|\nabla \psi_{l}\right|^{2}
$$

where infinitesimal variation gives:

$$
\begin{aligned}
& 2 \int d x\left\{z_{t t} \psi_{l}\left(\nabla \psi_{t}\right) \nabla \delta \chi(x)-z_{l l} \psi_{t}\left(\nabla \psi_{l}\right) \nabla \delta \chi(x)\right\} \\
&+2\left(z_{t t}-z_{l l}\right) \int d x\left(\nabla \psi_{t}\right)\left(\nabla \psi_{l}\right) \delta \chi(x) .
\end{aligned}
$$

The global gauge invariance of the action [with $\chi(x)=\chi$ ] gives the condition $z_{l l}=z_{t t}$. In this case, the resulting variation of the action reads:

$$
2 z_{t t} \int d x\left\{\left(\psi_{l} \nabla \psi_{t}\right)-\left(\psi_{t} \nabla \psi_{l}\right)\right\} \nabla \chi(x)
$$

that corresponds to the field coupled linearly to $\mathbf{A}(\mathbf{x})$ in Eq. (2.1).

In this way, we have clarified the precise meaning of local gauge invariance in terms of the new couplings. These results can be used, in particular, to determine the composite-field insertions of Appendix B.

\section{APPENDIX B: WARD IDENTITIES AT ONE-LOOP LEVEL}

In this Appendix, we compute explicitly the one-loop contribution of relevant vertex functions. We can thus analyze the validity of the WI through an explicit example. In particular, the cancellations of the divergent contribution in the WI will be demonstrated.

We begin with the analysis of the WI (3.12) when the coupling $v_{l}$ is nonvanishing. We will start by considering all running couplings present in the original action (2.1). The one-loop diagrams contributing to $\Gamma_{l}$ and $\Gamma_{t t}$ are:

$$
\begin{aligned}
\ldots & =-\frac{1}{2} v_{l t t} \sum_{q} \mathcal{G}_{t t}(q), \\
& =-\frac{1}{2} v_{l l t} \sum_{q} \mathcal{G}_{l l}(q), \\
& =-\frac{1}{2} v_{t t t t} \sum_{q} \mathcal{G}_{t t}(q), \\
& =-\frac{1}{2} v_{l l t t} \sum_{q} \mathcal{G}_{l l}(q), \\
& =-v_{l t t}^{2} \sum_{q}^{2} \mathcal{G}_{l l}(k-q) \mathcal{G}_{t t}(q)
\end{aligned}
$$


For non-vanishing $v_{l}$ the conditions on the bare running couplings can be obtained from the WI (3.12), (3.13), and (3.9) (once Fourier transformed). They give, respectively:

$$
\begin{aligned}
\psi_{l o} v_{t t}-v_{l} & =0, \\
v_{l t t} \psi_{l o}+v_{t t}-v_{l l} & =0, \\
v_{l l t t} \psi_{l o}+2 v_{l t t}-v_{l l l} & =0,
\end{aligned}
$$

while Eq. (3.19) is not modified (cf. Eq. (3.15)). The validity of Eq. (3.12) at the one-loop order can be now verified. Since $\Gamma_{l}$ is the sum of $v_{l},(\mathrm{~B} 1)$, and (B2), while $\Gamma_{t t}$ is the sum of $v_{t t}$ and (B3)-(B6), we can readily evaluate Eq. (3.12) for vanishing external momentum. The lowest order is clearly satisfied by the bare values $v_{l}=-2 \alpha\left(\mu-v \alpha^{2}\right)$ and $v_{t t}=-2\left(\mu-v \alpha^{2}\right)$. To verify that also the next order (one-loop) is correct, we can use the following identity

$$
\mathcal{G}_{l l}(q) \mathcal{G}_{t t}(q)-\mathcal{G}_{l t}(q) \mathcal{G}_{t l}(q)=-\frac{\mathcal{G}_{l l}(q)-\mathcal{G}_{t t}(q)}{v_{t t}-v_{l l}}
$$

holding for $u_{t t}=0$ and $z_{t t}=z_{l l}$. Adding all the contributions and using (B10), one can verify that the WI hold exactly.

However, in obtaining a renormalizable theory, all the irrelevant running couplings have been discarded (for instance, $v_{l l l}$ or $\left.v_{l l t t}\right)$. As anticipated in Section III A, the WI are invalidated by this fact but they continue to hold at the leading order for $k \rightarrow 0$. This can be verified by considering the contributions to $\Gamma_{l}$ and $\Gamma_{t t}$ when the marginal running couplings are set to zero. In this case, the contributions are (B1),(B3), (B5), and (B6), and the WI is not satisfied. We cannot test directly on this WI the cancellation of the leading divergent terms, since $\Gamma_{l}$ and $\Gamma_{t t}(0)$ are finite. One could, however, verify that the ultraviolet cutoff divergence is still perfectly balanced in the WI; more instructive examples can be obtained by looking at higher-order WI.

Let us consider accordingly the one-loop contributions to $\Gamma_{l l}, \Gamma_{l t t}$, and $\Gamma_{t t t t}$ due to the marginal couplings (from now on we set to zero all the irrelevant running couplings). On the same footing, the Green's functions are given by the expression (2.36) with $z_{l l}=0$, and $u_{t t}$ in general nonvanishing. The mean-field condition is applied to the lowest-order values for $v_{l t}$ and $v_{t t}$, which thus vanish. Notice that, using the above conditions, (B10) becomes:

$$
\mathcal{G}_{t t}(q) \mathcal{G}_{l l}(q)-\mathcal{G}_{l t}(q) \mathcal{G}_{t l}(q)=-\frac{\mathcal{G}_{t t}(q)}{v_{l l}}
$$

The one-loop diagram contributing to $\Gamma_{l l}(k)$ gives:

$$
\text { - - }-\gamma_{-}=-\frac{v_{l t t}^{2}}{2} \sum_{q} \mathcal{G}_{t t}(k-q) \mathcal{G}_{t t}(q) \text {. }
$$

We evaluate also the one-loop contributions to $\Gamma_{l t t}\left(k_{1}, k_{2}, k_{3}\right)$. For simplicity, we write only the value of the diagrams for vanishing external momenta, since the value for finite $k$ can be reconstructed from the following expressions by inserting the correct momenta in each internal Green's functions and adding the contribution of the permutations of the external arguments. We report below all the one-loop diagrams for $\Gamma_{l t t}$ :

$$
=v_{l t t}^{3} \sum_{q} \mathcal{G}_{t l}(q)^{2} \mathcal{G}_{t t}(q),
$$

Only in diagram (B14) there are two different permutations. With the same notation, we consider now the one-loop contributions to $\Gamma_{t t t t}$. We write the following equation:

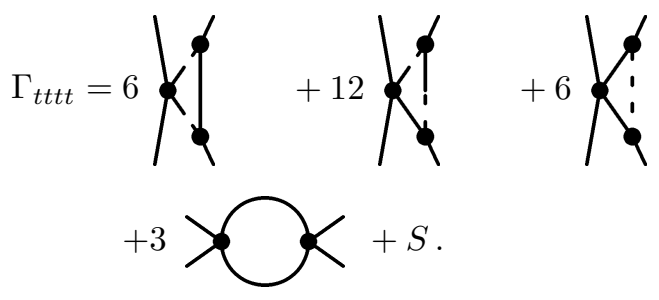

The numbers that multiply the diagrams indicate the non-equivalent permutations of the external $k_{i}$. The contribution of these diagrams is identical to the one given in (B13)-(B16), apart from the number of permutations and an overall factor $v_{t t t t} / v_{l t t}$ to be multiplied. Instead $S$ represents the sum of the following set of diagrams:

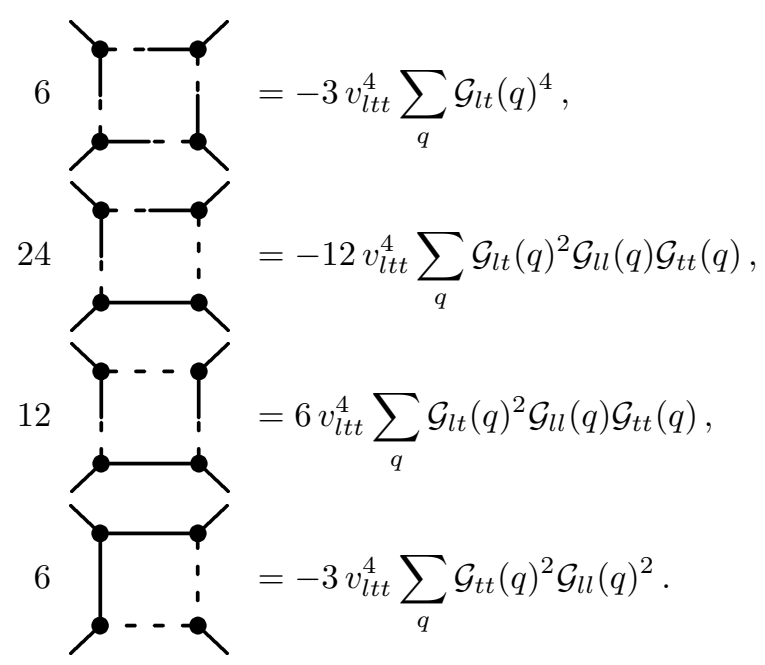


Adding all these contributions together and using (B11), we finally obtain for the $\Gamma$ functions at vanishing external $k$ :

$$
\begin{aligned}
\Gamma_{l t t} & =-\frac{1}{2} \frac{v_{l t t}^{2}}{\psi_{l o}^{2}} \sum_{q} \mathcal{G}_{t t}(q)^{2}, \\
\Gamma_{t t t t} & =-\frac{3}{2} \frac{v_{l t t}^{2}}{\psi_{l o}} \sum_{q} \mathcal{G}_{t t}(q)^{2} .
\end{aligned}
$$

The above expressions are divergent when $d \leq 3$. An IR cutoff should thus be introduced, with the vertex functions diverging when this cutoff goes to zero. It is readily verified that the leading diverging contributions of each vertex function simplify exactly when (B22), (B23), (B12), and (B3) are inserted into (3.13) and (3.15). Note, however, that there exist finite terms (specifically, the contribution from $\Gamma_{t t}$ ) that do not vanish, so that the WI is not exactly satisfied. This fact had to be expected and does not spoil the RG identity among the running couplings, since within the scheme of the dimensional renormalization they are valid for the diverging contributions. The PT is thus preserving the gauge invariance at the leading order in the IR divergences.

We consider now Eq. (3.11) as the simplest example of cancellation of the diverging coefficients of the external frequency $k_{o}$. To verify this, we need to evaluate $\Gamma_{l t}$ and $\Gamma_{l ; 0}$ for finite $k_{o}$. The one-loop contribution is:

$$
\begin{aligned}
& \Gamma_{l t}(k)=--\left\lceil\zeta=-v_{l t t}^{2} \sum_{q} \mathcal{G}_{t t}(k-q) \mathcal{G}_{l t}(q),\right. \\
& \Gamma_{l ; 0}(k)=--\bigcirc \sim=-\frac{1}{2} v_{l t t} w_{l t} \sum_{q} \mathcal{G}_{t t}(k-q) \mathcal{G}_{t t}(q) \text {. }
\end{aligned}
$$

When we evaluate Eq. (3.11) for $k_{i}=0$ and finite $k_{0}$, we obtain (we have used $\mathcal{G}_{l t}(k)=\mathcal{G}_{t t}(k) k_{0} w_{l t} / v_{l l}$ from (2.36)):

$$
-\frac{1}{2} v_{l t t} w_{l t} \sum_{q} \mathcal{G}_{t t}(k-q) \mathcal{G}_{t t}(q)\left(q_{o}-k_{o} / 2\right) .
$$

In order to extract the $k_{0}$ coefficient, let us perform the change of variables $q=q^{\prime}+k / 2$. Since an ultraviolet cutoff $\Lambda$ is assumed, the above change of variable should change the limits of integrations. We neglect this fact, since the difference is of higher order in $q_{0} / \Lambda$, and we are interested in the $q_{0} \rightarrow 0$ limit. With the above change of variable, we readibly obtain that the leading contribution of (B26) vanishes.

It is not difficult to evaluate also $\Gamma_{l: i}\left(k_{o}, \mathbf{k}\right)$ and insert the result into Eq. (3.11). We can evaluate then all $\Gamma$ functions at finite values of $k_{o}$ and $k_{i}$. The above results remain valid for the first part of the WI, since no use was made of the fact that $\mathbf{k}_{i}$ was vanishing. However, the contribution of the vector part of the WI is sub-leading with respect the one calculated in (B26). To check the vanishing of the vector part, one should thus perform the above calculation by keeping also the next-to-leading order.

\section{APPENDIX C: ONE-LOOP RG EQUATIONS}

In the text we have shown that a single coupling is actually scaling independently. In this Appendix, we obtain the one-loop RG equations for the four marginal couplings entering the propagators $\left(v_{l l}, w_{l t}, u_{t t}\right.$, and $\left.z_{t t}\right)$ and verify that the exact constraints found in Section IV B are fulfilled. We need to calculate explicitly the one loop contributions to $\Gamma_{l l}$ [depicted in (B12)], $\Gamma_{l t}$ [depicted in (B5)], and $\Gamma_{t t}$ [depicted in (B5), and (B6)]. According to the general procedure established in Section II B no correction to $\alpha$ need to be considered. The integrals entering these expressions can be written in terms of the following integrals (when the expression for the propagators in the small-momentum limit is considered):

$$
I_{n, m}(k)=\int \frac{d^{d+1} q}{(2 \pi)^{d+1}} \frac{q_{o}^{n}\left(\mathbf{q}^{2}\right)^{m / 2}}{(k+q)^{2} q^{2}} .
$$

We thus have at the one loop order:

$$
\begin{aligned}
\Gamma_{l l}(k)= & v_{l l}-\frac{v_{l l}^{2}}{2 \psi_{l o}^{2} z_{t t}^{2}} I_{0,0}(k) \\
\Gamma_{l t}(k)= & w_{l t} k_{0}+\frac{w_{l t} v_{l l}}{\psi_{l o}^{2} z_{t t}^{2}} I_{1,0}(k) \\
\Gamma_{t t}(k)= & u_{t t} k_{0}^{2}+z_{t t} \mathbf{k}^{2}-\frac{1}{\psi_{l o}^{2} z_{t t}^{2}}\left[\left(u_{t t} v_{l l}+w_{l t}^{2}\right) I_{2,0}(k)\right. \\
& \left.+z_{t t} v_{l l} I_{0,2}(k)+w_{l t}^{2} k_{0} I_{1,0}(k)\right] .
\end{aligned}
$$

The $I_{n, m}$ integrals are performed by standard procedures: ${ }^{14}$

$$
\begin{aligned}
I_{f}(k) & =\frac{\Gamma(2)}{\Gamma(1)^{2}} \int \frac{d^{d+1} q}{(2 \pi)^{d+1}} \int_{0}^{1} d x \frac{f(q)}{\left[x(k+q)^{2}+(1-x) q^{2}\right]^{2}} \\
& =\frac{1}{2} \int_{0}^{1} d x \int \frac{d^{d+1} q}{(2 \pi)^{d+1}} \frac{f(q-k x)+f(-q-k x)}{\left[q^{2}+k^{2} x(1-x)\right]^{2}}
\end{aligned}
$$

where $f(q)=q_{o}^{n} \mathbf{q}^{m}$. One can now use the identity

$$
\int_{0}^{+\infty} \frac{x^{d} d x}{\left(1+x^{2}\right)^{\alpha}}=\frac{\Gamma((d+1) / 2)) \Gamma(\alpha-(d+1) / 2)}{2 \Gamma(\alpha)}
$$

to obtain the analytic expression to be continued to complex values of the variable $d$. As a matter of fact, the integral (C5) converges only for $d-2 \alpha>0$, but its analytic continuation given by the Euler $\Gamma$-functions on the right-hand side of (C5) is defined for any value of the complex variable $d$, except for a discrete set of points where simple poles are present. Dimensional regularization proceeds by subtracting these poles that in our case are in $1 /(3-d)=1 / \epsilon$. We thus expand the integrals in $\epsilon$ 
keeping only the leading order. This gives the following expressions for $\Gamma_{i j}$ (we have absorbed the phase-space factor $1 /\left(8 \pi^{2}\right)$ in the definition of the running couplings $v_{l l} \rightarrow v_{l l} / 8 \pi^{2}$ and $\left.w_{l t} \rightarrow w_{l t} / \sqrt{8 \pi^{2}}\right)$ :

$$
\begin{aligned}
& \Gamma_{l l}(k)=v_{l l}-\frac{v_{l l}^{2}}{2 \psi_{l o}^{2} z_{t t}^{2}}{\frac{\left(k^{2}\right)}{\epsilon}}^{\epsilon / 2} \frac{c}{c_{o}} \\
& \Gamma_{l t}(k)=w_{l t} k_{0}-\frac{w_{l t} v_{l l}}{2 \psi_{l o}^{2} z_{t t}^{2}} k_{0} \frac{\left(k^{2}\right)}{\epsilon}{ }^{\epsilon / 2}\left(\frac{c}{c_{o}}\right)^{2} \\
& \Gamma_{t t}(k)=u_{t t} k_{0}^{2}+z_{t t} \mathbf{k}^{2}+{\frac{w_{l t}^{2}}{2 \psi_{l o}^{2}} z_{t t}^{2}}_{\frac{\left(k^{2}\right)}{\epsilon}}^{\epsilon / 2}\left(\frac{c}{c_{o}}\right)^{3}
\end{aligned}
$$

where $c^{2}$ indicates the combination of running couplings forming the square of the sound velocity [cf. Eq. (3.38)]. As a first result, we note that no divergence appears in the $\mathbf{k}^{2}$ dependence. This implies that $z_{t t}$ does not scale, as demonstrated using the WI in Section III B. The RG equations obtained by minimal subtraction for the remaining three couplings read:

$$
\begin{aligned}
\rho \frac{d v_{l l}}{d \rho} & =\frac{v_{l l}^{2}(\rho)}{2 \psi_{l o}^{2}(\rho) z_{t t}^{2}} \frac{c(\rho)}{c_{o}} \\
\rho \frac{d w_{l t}}{d \rho} & =\frac{v_{l l}(\rho) w_{l t}(\rho)}{2 \psi_{l o}^{2}(\rho) z_{t t}^{2}}\left(\frac{c(\rho)}{c_{o}}\right)^{2} \\
\rho \frac{d u_{t t}}{d \rho} & =-\frac{w_{l t}^{2}(\rho)}{2 \psi_{l o}^{2}(\rho) z_{t t}^{2}}\left(\frac{c(\rho)}{c_{o}}\right)^{3}
\end{aligned}
$$

where $\psi_{l o}(\rho)=\psi_{l o}(1) \rho^{\epsilon / 2}$ due to its bare dimension. It is now possible to verify that the RG equations admit the invariants $w_{l t}(\rho) / v_{l l}(\rho)$ and $c^{2}(\rho)$, by simply performing the derivative with respect to $\rho$ and substituting the RG equations. This shows at the one-loop level the validity of the identities proved at all orders in Section IV B. These identities can be used to eliminate two out of the three running couplings. For the last one $\left(v_{l l}\right.$, for instance), we need to solve the RG equation explicitly. The solution is given and discussed in Section IV C.

${ }^{1}$ S. Beliaev, Sov. Phys. JETP 34, 289 (1958).

${ }^{2}$ N. Hugenholtz and D. Pines, Phis. Rev. 116, 489 (1959).

${ }^{3}$ N. Bogoliubov, J. Phys. 11, 23 (1947).

${ }^{4}$ J. Gavoret and P. Nozieres, Ann. Phys. (N.Y.) 28, 349 (1964).

${ }^{5}$ K. Huang and A. Klein, Ann. Phys. (N.Y.) 30, 203 (1964).

${ }^{6}$ F. De Pasquale, G. Jona-Lasinio, and E. Tabet, Ann. Phys. (N.Y.) 33, 381 (1965).
${ }^{7}$ P. Hohenberg and P. Martin, Ann. Phys. (N.Y.) 34, 291 (1965).

${ }^{8}$ A. Nepomnyashchy and Y. A. Nepomnyashchy, Sov. Phys. JETP Lett. 21, 1 (1975). Y. A. Nepomnyashchy and A. Nepomnyashchy, Sov. Phys. JETP 48, 493 (1978). Y. A. Nepomnyashchy, Sov. Phys. JETP 58, 6611 (1983). Y. A. Nepomnyashchy, Phys. Rev. B 46, 6611 (1992).

${ }^{9}$ V. N. Popov, Functional Integrals and Collective Excitations (Cambridge University Press, Cambridge, 1987).

${ }^{10}$ V. Popov and A. V. Seredniakov, Sov. Phys. JETP 50, 193 (1979).

11 A.Z. Patašinskij and V.L. Pokrovskij, Sov. Phys.-JETP 37, 733 (1974).

12 C. Castellani, C. Di Castro, F. Pistolesi, and G.C. Strinati, Phys. Rev. Lett, 78, 1612 (1997).

${ }^{13}$ W. Metzner and C. Di Castro, Phys. Rev. B 47, 16107 (1993). W. Metzner, C. Castellani, C. Di Castro, Adv. Phys. 47, 317 (1998).

${ }^{14}$ D.J. Amit, Field Theory, the Renormalization Group, and Critical Phenomena (World Scientific, Singapore, 1984).

${ }^{15}$ P. B. Weichman, Phys. Rev. B 38, 8739 (1988).

${ }^{16}$ G. Benfatto, in Constructive Results in Field Theory, Statistical Mechanics, and Condensed Matter Physics, Palaiseau, July 25-27, (1994). See also, G. Benfatto and G. Gallavotti, Renormalization Group (Princeton Univ. Press, 1995).

${ }^{17}$ For a recent review on field theory methods for interacting boson gas see for instance O.J. Andersen, condmat/0305138 (unpublished).

18 M. E. Fisher, N.N. Barber, and D. Jasnow, Phys. Rev. A, 8 1111, (1973).

${ }^{19}$ A neat application of the loop expansion has been used recently to calculate the quantum corrections at two-loops order for the ground state energy of a condensate Bose gas: E. Braaten and A. Nieto, Eur. Phys. J. B 11, 143 (1999).

${ }^{20}$ The noninteracting boson system, on the contrary, with fixed chemical potential is not well defined (as at $T=0$ and $\mu<0$ the system contains no particle). As a consequence, the perturbative expansion is not well defined. On the other hand, the organization of classes of diagrams on the basis of the IR divergences -as we do- seems to be a well defined procedure since it bypasses the expansion in the bare potential.

${ }^{21}$ Monomials linear in the frequency and containing fields of the same type vanish identically, as they represent total derivatives in the action.

22 One can show by power counting that in the variables phase and modulus all running couplings are irrelevant. This is equivalent to stating that no IR divergences appear in the theory.

${ }^{23}$ See, e.g., C. Domb and M.S. Green, eds., Phase Transitions and Critical Phenomena, Vol. VI (Academic Press, N.Y., 1976).

${ }^{24}$ S. Giorgini, L. Pitaevskii, and S. Stringari, Phys. Rev. B 46, 6374 (1992). 